\title{
APPARENT SLIP FOR AN UPPER CONVECTED MAXWELL FLUID
}

\author{
ANDREAS MÜNCH* BARBARA WAGNER ${ }^{\dagger}$, L. PAMELA COOK ${ }^{\ddagger}$, AND RICHARD R. BRAUN ${ }^{\ddagger}$
}

\begin{abstract}
In this study the flow field of a nonlocal, diffusive upper convected Maxwell (UCM) fluid with a polymer in a solvent undergoing shearing motion is investigated for pressure driven planar channel flow and the free boundary problem of a liquid layer on a solid substrate. For large ratios of the zero shear polymer viscosity to the solvent viscosity, it is shown that channel flows exhibit boundary layers at the channel walls. In addition, for increasing stress diffusion the flow field away from the boundary layers undergoes a transition from a parabolic to a plug flow. Using experimental data for the wormlike micelle solutions $\mathrm{CTAB} / \mathrm{NaSal}$ and $\mathrm{CPyCl} / \mathrm{NaSal}$, it is shown that the analytic solution of the governing equations predicts these signatures of the velocity profiles. Corresponding flow structures and transitions are found for the free boundary problem of a thin layer sheared along a solid substrate. Matched asymptotic expansions are used to first derive sharp-interface models describing the bulk flow with expressions for an apparent slip for the boundary conditions, obtained by matching to the flow in the boundary layers. For a thin film geometry several asymptotic regimes are identified in terms of the order of magnitude of the stress diffusion, and corresponding new thin film models with a slip boundary condition are derived.
\end{abstract}

Key words. Wormlike micelle solutions, thin-film approximation, sharp-interface limit, matched asymptotic expansions

AMS subject classifications. 76A05, 34E05, 76A20

1. Introduction. Slip at the liquid-solid interface is a common phenomenon when liquid polymer layers are sheared along solid substrates. On the micro- or nanoscale of the liquid bulk system, this condition can have important implications for the liquid flow structure. A well-documented example is a polymer film that dewets from a hydrophobically coated substrate. An effective boundary condition for such complex systems is often given in the form of a Navier-Slip condition, relating the lateral velocity along the substrate to the shear rate $u=b u_{z}$. The quantity $b$ denotes an apparent slip length and encodes an underlying mesoscopic mechanism. For entangled polymer melts dewetting from a monolayer of polymer chains grafted on a substrate, such a mechanism is given by a coil-stretch transition into a disentangled state having much lower Rouse friction, and thus apparent viscosity, within a very thin layer near the substrate as has been shown by Brochard \& De Gennes [6]. The underlying mesoscopic mechanisms are different for polymer-melt solid-substrate systems, some of which are described in the reviews by Lauga et al. [16] or in Léger [17].

For polymer solutions or dilute polymer emulsions, analysis of the motion of the polymer chains within the thin interfacial region between the solid and the polymer suggest higher shear rates and lower viscosity within the interfacial region leading to an apparent velocity discontinuity and hence to an apparent slip, as discussed in $[2,3,7]$.

Further extensions of these studies regarding polymer-polymer apparent slip can be found in [1]. For a large class of colloidal suspensions apparent slip as well as shear banding are discussed in the review by Ballesta et al. [4]. For other complex liquids such as wormlike micellar solutions, slip also may relate to the occurrence of shear banding, which is closely related to a plateau region in the shear stress versus shear rate flow curve, which has been examined for example [11, 15, 18, 24, 26, 27, 28].

In channel flow experiments these wormlike micellar solutions show a thin band of high shear rate flow near the channel walls with a plug-like flow in the remaining portion of the channel [19] and shown in simulations of the Vasquez, Cook, McKinley (VCM) model [8]. The VCM model is a non-linear two species model constructed to account for the breaking and reforming of the wormlike micelles. The solution in the high shear rate band near the wall contains primarily short micelles, while in the center of the channel, the distribution of micellar lengths is close to equilibrium. Thus the shear banding can be loosely thought of as evidence of phase demixing. To understand and quantify the emergence and magnitude of apparent slip and also the transitions in flow structure for polymer solutions, we focus here on a model system much simpler than the VCM model, and employ an UCM model with sress diffusion [9] in a water solvent.

We address first the pressure driven planar channel flow, which has been investigated in [8]. After we formulate the boundary value problem in Section 2, we derive, in Section 3, an exact solution to the governing equations showing that the flow stuctures and transition in velocity profiles are controlled by two

* Mathematical Institute, University of Oxford, Andrew Wiles Building, Woodstock Road, Oxford OX2 6GG, UK (andreas.muench@maths.ox.ac.uk).

†Institute of Mathematics, Technical University Berlin, Str. des 17. Juni 136, 10623 Berlin, Germany (bwagner@math.tuberlin.de), Weierstrass Institute, Mohrenstrasse 39, 10117 Berlin, Germany (wagnerb@wias-berlin.de).

${ }^{\ddagger}$ Department of Mathematical Sciences, University of Delaware, Newark DE 19716, USA, (cook@udel.edu, rjbraun@udel.edu). 
where

parameters, the ratio of the solvent viscosity to the zero shear rate polymer viscosity and the non-dimensional stress diffusion parameter.

We then extend this analysis to the free boundary problems of a liquid layer shearing along a solid substrate in Section 4. We exploit the boundary layer flow structure to derive a reduced sharp-interface model with an apparent slip boundary conditions using matched asymptotic expansions. These sharp-interface models with an apparent slip form the basis for the derivation of new thin-film models governing the shape of the free surface for moderate to large slip lengths. These models are discussed in Section 5 together with a linear stability analysis yielding multiple relaxation modes for the for the case of large stress diffusion.

We conclude with a discussion of apparent slip on related problems in the context of dewetting liquid bi-layers in Section 6.

2. Maxwell fluid with solvent and diffusion. For convenience, we discuss two-dimensional flows throughout this study. The governing equations are those of an UCM fluid with stress diffusion [8, 9]. These equations are also visible within the VCM model for wormlike micellar solutions [27] by assuming that only one species of micelles is present.

The spatial coordinates and velocity are given by $\mathbf{x}^{\prime}=\left(x^{\prime}, z^{\prime}\right)$ and $\mathbf{v}^{\prime}=\left(u^{\prime}, w^{\prime}\right)$, corresponding to the streamwise and cross-stream directions, respectively. Time is denoted with $t^{\prime}$. Primes denote dimensional variables. Conservation of mass requires

$$
\nabla^{\prime} \cdot \mathbf{v}^{\prime}=0 .
$$

For $D_{t^{\prime}}=\partial_{t^{\prime}}+u^{\prime} \partial_{x^{\prime}}+w^{\prime} \partial_{z^{\prime}}$, conservation of momentum can be written as

$$
\rho D_{t^{\prime}} \mathbf{v}^{\prime}=\nabla^{\prime} \cdot \mathbf{\Pi}^{\prime}
$$

$$
\mathbf{\Pi}^{\prime}=-\mathbf{p} \mathbf{I}+\eta_{\mathbf{s}} \dot{\gamma}^{\prime}-\tau_{\mathbf{p}}^{\prime}
$$

is the total stress, $\dot{\gamma}^{\prime}=\nabla^{\prime} \mathbf{v}^{\prime}+\left(\nabla^{\prime} \mathbf{v}^{\prime}\right)^{\mathbf{t}}$ the strain rate and the superscript $\mathbf{t}$ denotes the transpose. Here $73 \tau_{p}^{\prime}=-\mathbf{A}^{\prime}+G_{0} \mathbf{I}$ denotes the polymer stress, which is governed by

The density is denoted by $\rho$, the solvent viscosity by $\eta_{s}$, and the zero shear viscosity of the solution by $\eta_{0}$. The latter is the sum of the solvent viscosity and the contribution $\eta_{p}^{0}=G_{0} \lambda$ from the micelles, that is, $\eta_{0}=\eta_{s}+\eta_{p}^{0}$, where $\lambda$ is the relaxation time of the micelles and $G_{0}$ is the shear modulus at zero strain rate.

76 We denote the upper convected derivative of a quantity $\mathbf{f}$ by

$$
\mathbf{f}_{\left(1^{\prime}\right)}=D_{t^{\prime}} \mathbf{f}-\left(\nabla^{\prime} \mathbf{v}^{\prime}\right)^{\mathbf{t}} \cdot \mathbf{f}-\mathbf{f} \cdot\left(\nabla^{\prime} \mathbf{v}^{\prime}\right)
$$

The addition of the stress diffusion term is discussed in $[8,9]$. The boundary conditions for the problem are

and, because of the diffusion term, we also need boundary conditions on the stress. We assume no flux of

85 At the free surface $z^{\prime}=h^{\prime}$, we have

$$
\mathbf{v}^{\prime}=\mathbf{0}
$$

$$
\partial_{z^{\prime}} \mathbf{A}^{\prime}=0
$$

$$
\mathbf{v}^{\prime} \cdot \nabla^{\prime} F^{\prime}+\partial_{t^{\prime}} F^{\prime}=0
$$


where $F^{\prime}\left(x^{\prime}, h^{\prime}\left(x^{\prime}, t^{\prime}\right), t^{\prime}\right)=z^{\prime}-h^{\prime}\left(x^{\prime}, t^{\prime}\right)=0$ defines the location of the free surface. This results in the

kinematic condition

$$
\partial_{t^{\prime}} h^{\prime}-u^{\prime} \partial_{x^{\prime}} h-w^{\prime}=0
$$

The normal stress balance at the free surface is

$$
\left[\left[\mathbf{n}^{\prime} \cdot \boldsymbol{\Pi}^{\prime} \cdot \mathbf{n}^{\prime}\right]\right]=\kappa \sigma
$$

where $\kappa=\nabla^{\prime} \cdot \mathbf{n}$ is the curvature of the surface and $\sigma$ is the surface tension of the film/air interface. We define the jump in a function $f^{\prime}$ across the film/air interface as $\left[\left[f^{\prime}\right]\right]=f_{\text {air }}^{\prime}-f_{\text {film }}^{\prime}$. We further assume that the air is a passive gas with zero stress components and pressure. The tangential stress balance is given by

$$
\left[\left[\mathbf{t}^{\prime} \cdot \mathbf{\Pi}^{\prime} \cdot \mathbf{n}^{\prime}\right]\right]=0
$$

at the surface. Due to the inclusion of stress diffusion we need a boundary condition on stress at the free surface. Using the no flux boundary conditions of conformation gives

$$
\mathbf{n}^{\prime} \cdot \nabla^{\prime} \mathbf{A}^{\prime}=0
$$

on the free surface. In detail, the normal and tangential stress boundary conditions are, respectively,

$$
-p^{\prime}+\mathbf{n}^{\prime} \cdot\left(\mathbf{A}^{\prime}-G_{0} \mathbf{I}+\eta_{s} \dot{\gamma}\right) \cdot \mathbf{n}^{\prime}=\sigma \partial_{x^{\prime}}^{2} h^{\prime}\left(N^{\prime}\right)^{-3}
$$

and

$$
\begin{aligned}
{\left[1-\left(\partial_{x^{\prime}} h^{\prime}\right)^{2}\right]\left[A_{x z}+\eta_{s}\left(\partial_{z^{\prime}} u^{\prime}+\partial_{x^{\prime}} w^{\prime}\right)\right] } & -\partial_{x^{\prime}} h^{\prime}\left(A_{x x}^{\prime}+2 \eta_{s} \partial_{x^{\prime}} u^{\prime}\right) \\
& +\partial_{x^{\prime}} h^{\prime}\left(A_{z z}^{\prime}+2 \eta_{s} \partial_{z^{\prime}} w^{\prime}\right)=0 .
\end{aligned}
$$

We have made use of

$$
\mathbf{n}^{\prime}=\left(-\partial_{x^{\prime}} h^{\prime} \mathbf{i}+\mathbf{j}\right)\left(N^{\prime}\right)^{-1}, \quad \mathbf{t}^{\prime}=\left(\mathbf{i}+\partial_{x^{\prime}} h^{\prime} \mathbf{j}\right)\left(N^{\prime}\right)^{-1}, \quad \text { and } \quad N^{\prime}\left[1+\left(\partial_{x^{\prime}} h^{\prime}\right)^{2}\right]^{1 / 2}
$$

We non-dimensionalize the governing equations by setting

$$
x^{\prime}=\ell x, z^{\prime}=H z, h^{\prime}=H h, u^{\prime}=U u, w^{\prime}=\epsilon U w, t^{\prime}=\frac{\ell}{U} t, p^{\prime}=\frac{G_{0}}{\epsilon} p, \mathbf{A}^{\prime}=G_{0} \mathbf{A} .
$$

Here $H$ is the the characteristic thickness of the film, $\ell$ the characteristic length along it. $U$ the characteristic speed along the film. The following non-dimensional parameters arise:

$$
\epsilon=\frac{H}{\ell}, \operatorname{Re}=\frac{\rho U H}{\eta_{s}}, \operatorname{De}=\frac{\lambda U}{H}, \delta=\sqrt{\frac{D_{s} \lambda}{H^{2}}}, \quad \text { and } \alpha=\sqrt{\frac{\delta}{\eta_{p}^{0} / \eta_{s}}} \text {. }
$$

Re is the Reynolds number, De is the Deborah number, $\delta$ is the nondimensional stress diffusion parameter and $D_{t}=\partial_{t}+u \partial_{x}+w \partial_{z}$. We note that in this study, we scale the pressure larger than the polymer stress terms. However, other choices where pressure and polymer stress are of the same order may also become relevant. In the momentum equation we balance

$$
\frac{\alpha^{2}}{\delta} \operatorname{De} \partial_{z}^{2} u \sim \partial_{z} A_{x z} \sim \partial_{x} p
$$

For the derivation of the lubrication problem with a free boundary, we require that at the free boundary the pressure $p$ is balanced by surface tension. Hence, in the normal stress condition we let

$$
S_{p}=\frac{\sigma \epsilon^{3}}{G_{0} H}=O(1)
$$


which means that

$$
\frac{\sigma H}{\ell^{2}} \sim \frac{G_{0}}{\epsilon} \quad \text { or } \quad \epsilon^{3} \sim \frac{G_{0} H}{\sigma} .
$$

The nondimensional governing equations then become as follows. For conservation of mass,

$$
\partial_{x} u+\partial_{z} w=0
$$

For momentum conservation,

$$
\begin{array}{r}
\epsilon \frac{\alpha^{2}}{\delta} \operatorname{DeRe} D_{t} u=-\partial_{x} p+\frac{\alpha^{2}}{\delta} \operatorname{De}\left(\epsilon^{2} \partial_{x}^{2} u+\partial_{z}^{2} u\right)+\epsilon \partial_{x} A_{x x}+\partial_{z} A_{x z}, \\
\epsilon^{3} \frac{\alpha^{2}}{\delta} \operatorname{De} \operatorname{Re} D_{t} w=-\partial_{z} p+\epsilon^{2} \frac{\alpha^{2}}{\delta} \operatorname{De}\left(\epsilon^{2} \partial_{x}^{2} w+\partial_{z}^{2} w\right)+\epsilon\left(\epsilon \partial_{x} A_{x z}+\partial_{z} A_{z z}\right) .
\end{array}
$$

For the polymer part of the deviatoric stress, the equations are as follows:

$$
\begin{array}{r}
\epsilon \operatorname{De}\left(D_{t} A_{x x}-2 \epsilon^{-1} A_{x z} \partial_{z} u-2 \epsilon A_{x x} \partial_{x} u\right)+A_{x x}-1=\delta\left(\epsilon^{2} \partial_{x}^{2} A_{x x}+\partial_{z}^{2} A_{x x}\right), \\
\epsilon \operatorname{De}\left(D_{t} A_{x z}-\epsilon^{-1} A_{z z} \partial_{z} u-\epsilon A_{x x} \partial_{x} w\right)+A_{x z}=\delta\left(\epsilon^{2} \partial_{x}^{2} A_{x z}+\partial_{z}^{2} A_{x z}\right), \\
\epsilon \operatorname{De}\left(D_{t} A_{z z}-2 A_{z z} \partial_{z} w-2 \epsilon A_{x z} \partial_{x} w\right)+A_{z z}-1=\delta\left(\epsilon^{2} \partial_{x}^{2} A_{z z}+\partial_{z}^{2} A_{z z}\right) .
\end{array}
$$

The boundary conditions are, at $z=0$,

$$
u=w=0 \text { and } \partial_{z} A_{i j}=0,
$$

with $i=x, z$ and $j=x, z$. For the free surface boundary conditions at $z=h(x, t)$ we have:

$$
\begin{aligned}
& \partial_{t} h-u \partial_{x} h=w \\
& -S_{p} \partial_{x}^{2} h N^{-3}=p+N^{-2}\left\{-\epsilon\left[A_{z z}-1+\epsilon^{2}\left(\partial_{x} h\right)^{2}\left(A_{x x}-1\right)\right]\right. \\
& -2 \frac{\alpha^{2}}{\delta} \epsilon^{2} \operatorname{De} \partial_{x} h\left(\partial_{z} u+\epsilon^{2} \partial_{x} w\right) \\
& \left.-2 \epsilon \partial_{x} h A_{x z}+2 \frac{\alpha^{2}}{\delta} \epsilon^{2} \operatorname{De}\left[\partial_{z} w+\epsilon^{2}\left(\partial_{x} h\right)^{2} \partial_{x} u\right]\right\}, \\
& 0=\epsilon \partial_{x} h\left(A_{z z}-A_{x x}\right)+A_{x z}\left[1-\epsilon^{2}\left(\partial_{x} h\right)^{2}\right]+\frac{\alpha^{2}}{\delta} \operatorname{De}\left(\partial_{z} u+\epsilon^{2} \partial_{x} w\right)\left[1-\epsilon^{2}\left(\partial_{x} h\right)^{2}\right] \\
& -2 \frac{\alpha^{2}}{\delta} \operatorname{De} \epsilon \partial_{x} h\left(\partial_{x} u-\partial_{z} w\right) \text {, }
\end{aligned}
$$

and

$$
\left(\partial_{z} A_{i j}-\epsilon^{2} \partial_{x} h \partial_{x} A_{i j}\right) N^{-1}=0 .
$$

We have used

$$
\mathbf{n}=\left(-\epsilon \partial_{x} h \mathbf{i}+\mathbf{j}\right) N^{-1}, \quad \mathbf{t}=\left(\mathbf{i}+\epsilon \partial_{x} h \mathbf{j}\right) N^{-1}, \quad \text { and } \quad N=\left[1+\left(\epsilon \partial_{x} h\right)^{2}\right]^{1 / 2}
$$

as well as

$$
\overline{\mathrm{Ca}}=\eta_{0} U / \sigma \epsilon^{3} \text { and } S_{p}=\sigma \epsilon^{2} / G_{0} \ell=\mathrm{De} / \overline{\mathrm{Ca}} .
$$


3. Boundary layers in planar channel flow. To study the effect of the parameters $\alpha$ and $\delta$, we first consider the problem of planar channel flow, where we can find explicit solutions [8]. In this case, we normalize with the width of the channel $h$ so that the cross-stream variable is $-1 / 2<z<1 / 2$. The boundary conditions at each side wall $(z= \pm 1 / 2)$ are those of no slip, $u=0$, and no flux of conformation/stress, $\partial_{z} A_{i j}=0$ with $i=x, z$ and $j=x, z$. Consistent with parallel shear flow, we assume that $w=0$ and that $\partial_{x} p$ and all other variables are independent of $x$. We then obtain from (2.4f) and (2.4g) that $A_{z z}=1$. Using this in (2.4e), solving for $\partial_{z} u$, inserting the result into $(2.4 \mathrm{~b})$ and integrating the latter once, we obtain

$$
\frac{\alpha^{2}}{1+\alpha^{2} / \delta} \partial_{z}^{2} A_{x z}-A_{x z}=-\frac{1}{1+\alpha^{2} / \delta}(z-c) \partial_{x} p .
$$

The constant of integration $c=0$ can be set to zero if we assume the flow field is symmetric about $z=0$, that is, $u$ is an even function in $z$. Integrating (3.1) and using (2.4g) gives

$$
A_{x z}=\frac{\partial_{x} p}{1+\alpha^{2} / \delta}\left[\hat{z}-\frac{\alpha}{\sqrt{1+\alpha^{2} / \delta}} \frac{\sinh \left(\sqrt{1+\alpha^{2} / \delta} \frac{\hat{z}}{\alpha}\right)}{\cosh \left(\sqrt{1+\alpha^{2} / \delta} \frac{1}{2 \alpha}\right)}\right] .
$$

Substituting into the first integral of the momentum equation gives the velocity component along the channel:

$$
u=-\frac{\partial_{x} p}{\operatorname{De}} \frac{1}{1+\alpha^{2} / \delta}\left(T_{1}+T_{2}\right)
$$

where

$$
T_{1}=-\left(\frac{\hat{z}^{2}}{2}-\frac{1}{8}\right), \quad \text { and } \quad T_{2}=\frac{\delta}{1+\alpha^{2} / \delta}\left[1-\frac{\cosh \left(\sqrt{1+\alpha^{2} / \delta} \frac{\hat{z}}{\alpha}\right)}{\cosh \left(\sqrt{1+\alpha^{2} / \delta} \frac{1}{2 \alpha}\right)}\right]
$$

This velocity distribution can develop boundary layers and plug flow depending on the sizes of $\alpha$ and $\delta$. To see this, we consider the two terms $T_{1}$ and $T_{2}$ in (3.3) for fixed channel width. First consider $\alpha$ and the no-slip boundary condition; a boundary layer occurs when $\alpha$ in the cosh's in $T_{2}$ is small,

$$
\alpha \ll 1 .
$$

For $\alpha \ll 1$ and $|z|<1 / 2$ fixed, $T_{2} \rightarrow \delta$ which will not satisfy a no-slip boundary condition. On the other hand, $\hat{z} \rightarrow \pm 1 / 2$ for fixed $\alpha$ leads to $T_{2} \rightarrow 0$, which does satisfy the no-slip requirement; the different limits imply that boundary layers occur in $T_{2}$ at $z= \pm 1 / 2$. The transition to plug-flow behavior is possible if the parabolic velocity profile from the first term $T_{1}$ does not contribute significantly to the flow, which is the case if $\delta \gg 1$.

Fig. 1 shows the flow field $u$ for several choices of $\delta$ and $\alpha$; we normalized with its maximum for ease of comparison. Fig. 1 shows plots for $\delta=0.1,1$ and 10; in each plot, $u$ is plotted for three different values of $\alpha$ (dotted, $2.2 \times 10^{-2}$; dashed, $7.1 \times 10^{-3}$; solid, $2.2 \times 10^{-3}$ ). In all cases $u=0$ at the boundary, but as $\alpha$ decreases, a boundary layer becomes more apparent at each value of $\delta$. The insets shows an enlarged view of the plots near $z=1 / 2$, and it is clearly seen that a boundary layer develops as $\alpha$ decreases. In particular in the inset of the middle and the figure on the bottom, the width of the layer decreases by about the same factor as $\alpha$ and is in fact largely independent of $\delta$ as expected from $T_{2}$ in (3.3). The boundary layers in the left sub-figure are less obvious as the parabolic contribution $T_{1}$, which does not have boundary layers of its own, dominates the appearance of the flow profile for small $\delta . \delta>0$ is needed to have boundary layers, and therefore these layers only develop in the presence of stress diffusion. We note that fixed $\delta$ with decreasing $\alpha$ implies increasing the polymer-to-solvent viscosity ratio $\eta_{p}^{0} / \eta_{s}$. 

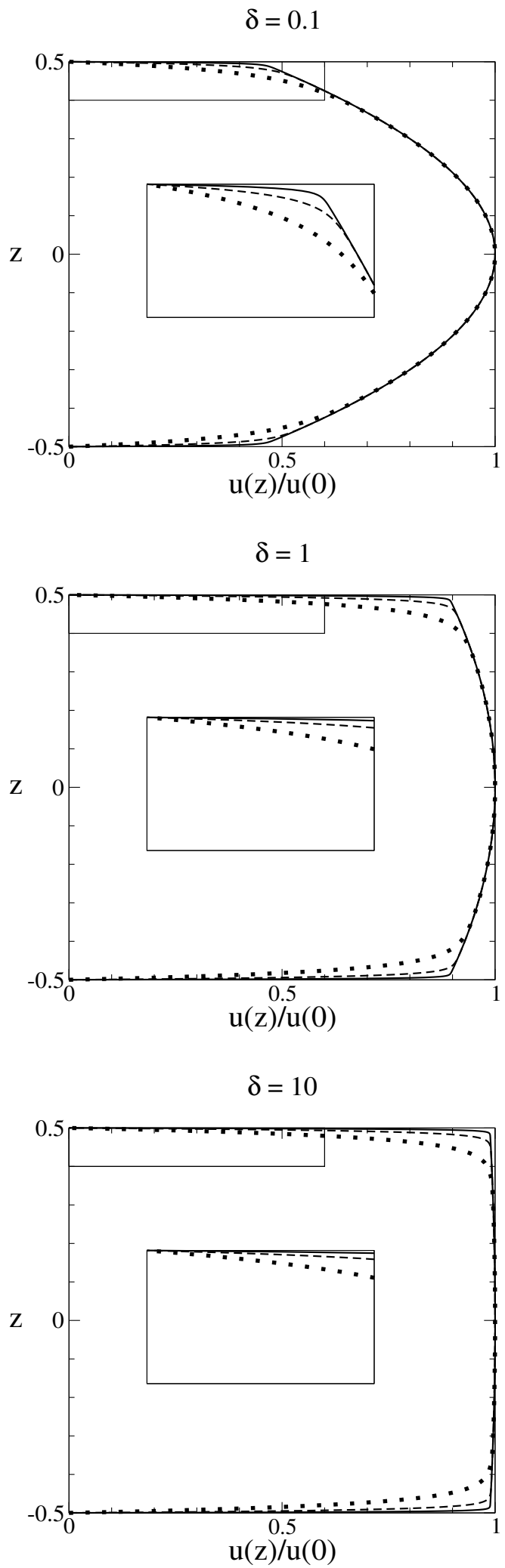

FIG. 1. Velocity profiles for different $\delta=0.1,1,10$ as shown in the title from top to bottom, and for different $\alpha=2.2 \times 10^{-2}$, $7.1 \times 10^{-3}, 2.2 \times 10^{-3}$, shown in each sub-figure by a dotted, dashed and solid line, respectively. For clarity, we enlarged in each ubfigure the boundary layer region in an inset. 
We now compare velocity profiles for the same $\alpha$ but different $\delta$, that is, for $\delta=0.1,1$ and 10 . We observe that the parabolic profile that is present for the smaller $\delta$ flattens out for $\delta=1$ and becomes a plug-flow profile for $\delta=10$, or more generally, for large $\delta$. This plug flow only develops with substantial stress diffusion across the channel. Indeed, $\delta \gg 1$ implies $H \ll \sqrt{D_{s} \lambda}$ (see (2.3)). For the physical parameters obtained from [5] and given in Table 1 and its caption, $\sqrt{D_{s} \lambda}$ is in the range of $10 \ldots 100 \mu \mathrm{m}$ and therefore plug-flow situations are only relevant for channel widths of tens of microns or smaller. Thus we emphasize that both the formation of boundary layers and the transition to plug flow is linked to the presence of stress diffusion.

As an example we show some typical parameter values for $\mathrm{CTAB} / \mathrm{NaSal}$ and $\mathrm{CPyCl} / \mathrm{NaSal}$ solutions of different concentrations and channel widths in Table 1 and the corresponding velocity profiles in Fig. 2. Again, the results are scaled to have unit size flow domain in $|z|<1 / 2$ (the actual channel width $H$ enters through the non-dimensional parameters) and normalised by $u(0)$. For each solution and concentration, the profiles are shown for two choices of $H$. Since both $\delta$ and $\alpha$ increase with $H$, the curvature of the profile near the center of the channel and the width of the boundary layer change simultaneously, with the more plug-like flow and wider boundary layers occurring for the smaller $H$. The profiles typically have a visible curvature, with a distinctive plug flow behaviour with thin boundary layers occuring only for the largest $\delta$ in table 1 , see rows 3 (corresponds to rightmost solid line in left sub-figure) and 8 (corresponds to rightmost dashed line in the right sub-figure). Notice that the relaxation time $\lambda$ decreases with concentration for $\mathrm{CPyCl} / \mathrm{NaSal}$ but decreases dramatically for $\mathrm{CPyCl} / \mathrm{NaSal}$, and therefore the trends in $\delta$ are also reversed.

Returning to consider a fixed $\delta>0$, we note that the asymptotic structure of the flow for $\alpha$ can be used to interpret the effect of $\delta$ as a slip length on the outer solution i.e. at an $O(1)$ distance from the walls. The leading order outer solution is obtained by taking the limit $\alpha \rightarrow 0$ in (3.3) for fixed $\delta$ and $z$, with $|z|<1 / 2$, giving

$$
u=-\frac{\partial_{x} p}{\operatorname{De}}\left\{-\left(\frac{\hat{z}^{2}}{2}-\frac{1}{8}\right)+\delta\right\} .
$$

Interestingly, the resulting flow profile has the same parabolic form as for a Newtonian flow in a channel except that it does not satisfy the no-slip condition at $z= \pm 1 / 2$. The finite slip velocity at the walls can be used to define a slip length via the Navier-slip condition

$$
\left.\frac{|u|}{\left|u_{z}\right|}\right|_{z= \pm 1 / 2}=2 \delta
$$

suggesting a slip length of $2 \delta$.

If we now consider the sub-limit $\delta \gg 1$, we expect to see the shear stress at the wall to drop to zero and thus the development of plug flow. Indeed, if we rescale $u=\delta \bar{u}$, we get in the limit $\delta \rightarrow \infty$ that

$$
\bar{u}=-\frac{\partial_{x} p}{\mathrm{De}}
$$

which is constant in $z$.

For this upper convected Maxwell model, we have shown that the limit of small $\alpha$, corresponding to small solvent viscosity, results in boundary layers at the walls of the channel. Furthermore, for large $\delta$, corresponding to thin films relative to the polymer stress diffusion length, plug flow develops in the interior of the channel. We now move on to consider a thin film with a deforming free surface and use these results to approximate the velocity field and polymer stress distribution inside the flowing fluid. With those approximate flow and stress fields, we derive equations for the film thickness and leading order polymer stress in different limits.

4. Sharp-interface limit for the free boundary flow. We now consider flow on the domain $0 \leq$ $z \leq h(x, t)$ with overall dimensional thickness $H$ as before. The free surface at $z=h$ must be found as part of the problem as is typical, and there is still a no-slip and impenetrable substrate at $z=0$. We derive a sharp-interface model in the limit $\alpha \rightarrow 0$ for the scaled full governing equations (2.4) including the free boundary at $z=h$, leaving $\varepsilon$ and $\delta$ fixed. This approach leads to an outer problem for which matching to the bottom boundary layer at the substrate at $z=0$ results in a Navier-slip-like condition. The leading order outer problem can then be passed through the thin film limit $\varepsilon \rightarrow 0$ in the following section, with different cases for the different regimes of $\delta$. 


\begin{tabular}{|l|c|c|c|c|c|c|c|c|c|c|}
\hline CTAB/NaSal & $G_{0}$ & $\lambda$ & $\eta_{0}$ & $U$ & $S_{p}$ & Re & De & $H$ & $\delta$ & $\alpha$ \\
\hline $25 / 25$ & 2.5 & 27 & 68 & $1.5 \mathrm{e}-7$ & $1.5 \mathrm{e}-6$ & $1.5 \mathrm{e}-6$ & $4.0 \mathrm{e}-2$ & $1 \mathrm{e}-4$ & 1.6 & $4.9 \mathrm{e}-3$ \\
$150 / 150$ & 94.8 & 0.4 & 38 & $2.6 \mathrm{e}-7$ & $2.6 \mathrm{e}-6$ & $2.6 \mathrm{e}-6$ & $1.1 \mathrm{e}-3$ & $1 \mathrm{e}-4$ & $2.0 \mathrm{e}-1$ & $2.3 \mathrm{e}-3$ \\
\hline $25 / 25$ & 2.5 & 27 & 68 & $1.5 \mathrm{e}-7$ & $1.5 \mathrm{e}-6$ & $1.5 \mathrm{e}-6$ & $4.0 \mathrm{e}-1$ & $1 \mathrm{e}-5$ & 16 & $1.6 \mathrm{e}-2$ \\
$150 / 150$ & 94.8 & 0.4 & 38 & $2.6 \mathrm{e}-7$ & $2.6 \mathrm{e}-6$ & $2.6 \mathrm{e}-6$ & $1.1 \mathrm{e}-2$ & $1 \mathrm{e}-5$ & 2 & $7.3 \mathrm{e}-3$ \\
\hline \hline $\mathrm{CPyCl} / \mathrm{NaSal}$ & $G_{0}$ & $\lambda$ & $\eta_{0}$ & $U$ & $S_{p}$ & Re & De & $H$ & $\delta$ & $\alpha$ \\
\hline $50 / 25$ & 4.2 & $7.7 \mathrm{e}-1$ & 2.8 & $3.5 \mathrm{e}-6$ & $3.1 \mathrm{e}-5$ & $3.1 \mathrm{e}-5$ & $2.7 \mathrm{e}-2$ & $1 \mathrm{e}-4$ & $2.8 \mathrm{e}-1$ & $9.9 \mathrm{e}-3$ \\
$200 / 100$ & 100 & 1.7 & 200 & $5.1 \mathrm{e}-8$ & $5.7 \mathrm{e}-7$ & $5.7 \mathrm{e}-7$ & $8.6 \mathrm{e}-4$ & $1 \mathrm{e}-4$ & $4.1 \mathrm{e}-1$ & $1.4 \mathrm{e}-3$ \\
\hline $50 / 25$ & 4.2 & $7.7 \mathrm{e}-1$ & 2.8 & $3.5 \mathrm{e}-6$ & $3.1 \mathrm{e}-5$ & $3.1 \mathrm{e}-5$ & $2.7 \mathrm{e}-1$ & $1 \mathrm{e}-5$ & 2.8 & $3.1 \mathrm{e}-2$ \\
$200 / 100$ & 100 & 1.7 & 200 & $5.1 \mathrm{e}-8$ & $5.7 \mathrm{e}-7$ & $5.7 \mathrm{e}-7$ & $8.6 \mathrm{e}-3$ & $1 \mathrm{e}-5$ & 4.1 & $4.6 \mathrm{e}-3$ \\
\hline
\end{tabular}

Parameter values for $\mathrm{CTAB} / \mathrm{NaSal}$ (top) and for $\mathrm{CPyCl} / \mathrm{NaSal}$ (bottom) solutions at different concentrations in $\mathrm{mM}$ and for two layer thicknesses $H$. The values are obtained from A. Bhardwaj, E. Miller, and J. P. Rothstein, J of Rheology, 51:693719 (2007) [5]. The units for $\lambda, U$ and $H$ are $s$ and $\mathrm{m} / \mathrm{s}$ and $m$, respectively. Here $\rho=10^{3} \mathrm{~kg} / \mathrm{m}^{3}$ and $U$ is chosen by making $\overline{C a}=1, D_{s}=10^{-9} \mathrm{~m}^{2} / \mathrm{s}$ and $\eta_{s}=10^{-3} \mathrm{~kg} / \mathrm{ms}$. Note that $\overline{C a}=1$ enforces $S_{p}=$ Re.
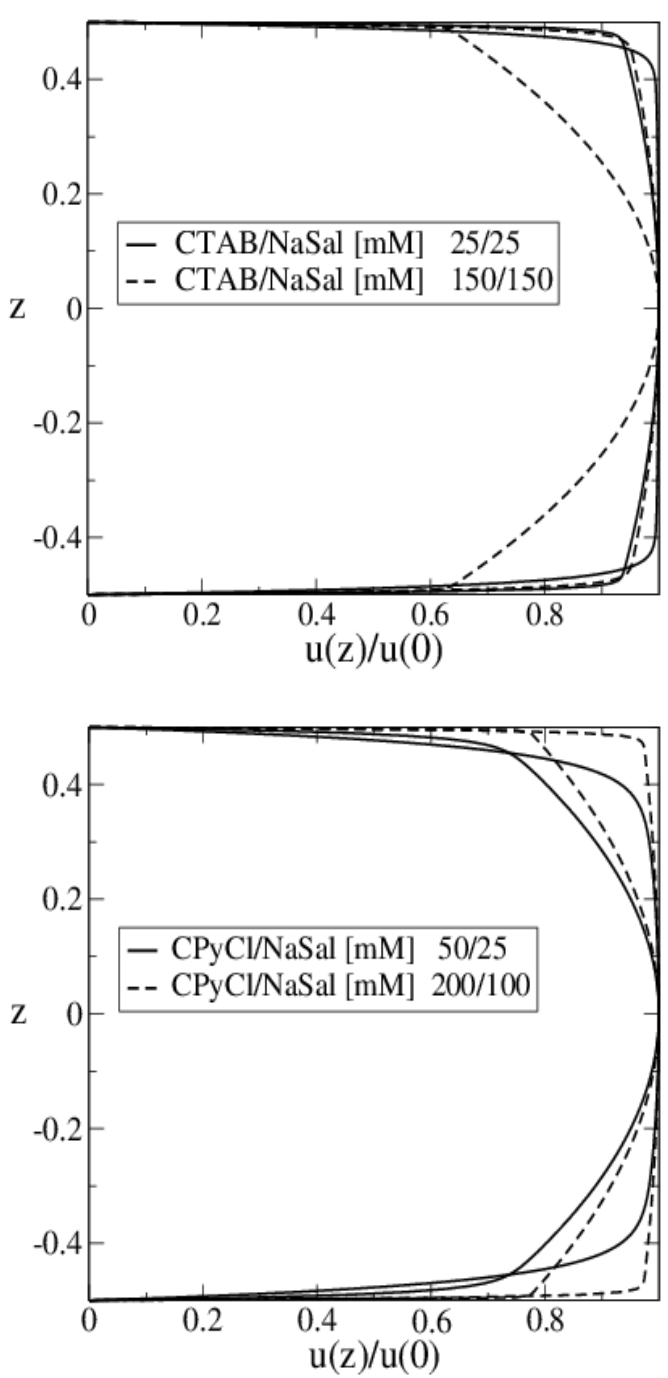

FIG. 2. Velocity profiles for different ratios concentrations of CTAB/NaSal and CPyCl/NaSal solutions and channel widths as given in Table 1. $H$ is smaller for the velocity profiles that are closer to plug flow near $z=0$. 
4.1. Outer problem. We first treat the flow away from the boundaries. We assume the outer variables depend on $x, z$ and $t$, and that they can be expanded in a regular expansion in $\alpha$ :

$$
\begin{gathered}
u=u^{(0)}+\alpha u^{(1)}+\cdots, \quad p=p^{(0)}+\alpha p^{(1)}+\cdots \\
w=w^{(0)}+\alpha w^{(1)}+\cdots, \quad A_{i j}=A_{i j}^{(0)}+\alpha A_{i j}^{(1)}+\cdots
\end{gathered}
$$

Then, to leading order in $\alpha$ mass and momentum conservation become

$$
\begin{aligned}
& 0=\partial_{x} u^{(0)}+\partial_{z} w^{(0)} \\
& 0=-\partial_{x} p^{(0)}+\epsilon \partial_{x} A_{x x}^{(0)}+\partial_{z} A_{x z}^{(0)}, \\
& 0=-\partial_{z} p^{(0)}+\epsilon\left(\epsilon \partial_{x} A_{x z}^{(0)}+\partial_{z} A_{z z}^{(0)}\right) .
\end{aligned}
$$

For the polymer part of the deviatoric stress, the equations are as follows:

$$
\begin{array}{r}
\epsilon \operatorname{De}\left(D_{t} A_{x x}^{(0)}-2 \epsilon^{-1} A_{x z}^{(0)} \partial_{z} u^{(0)}-2 A_{x x}^{(0)} \partial_{x} u^{(0)}\right)+A_{x x}^{(0)}-1=\delta\left(\epsilon^{2} \partial_{x}^{2} A_{x x}^{(0)}+\partial_{z}^{2} A_{x x}^{(0)}\right), \\
\epsilon \operatorname{De}\left(D_{t} A_{x z}^{(0)}-\epsilon^{-1} A_{z z}^{(0)} \partial_{z} u^{(0)}-\epsilon A_{x x}^{(0)} \partial_{x} w^{(0)}\right)+A_{x z}^{(0)}=\delta\left(\epsilon^{2} \partial_{x}^{2} A_{x z}^{(0)}+\partial_{z}^{2} A_{x z}^{(0)}\right), \\
\epsilon \operatorname{De}\left(D_{t} A_{z z}^{(0)}-2 A_{z z}^{(0)} \partial_{z} w^{(0)}-2 \epsilon A_{x z}^{(0)} \partial_{x} w^{(0)}\right)+A_{z z}^{(0)}-1=\delta\left(\epsilon^{2} \partial_{x}^{2} A_{z z}^{(0)}+\partial_{z}^{2} A_{z z}^{(0)}\right) .
\end{array}
$$

The boundary conditions at $z=0$ and $z=h$ need to be obtained from matching to solutions of appropriate boundary layer problems.

4.2. Inner problem at the substrate. At $z=0$ the highest $z$-derivatives of $u$ have dropped out. For the boundary layer there, we introduce the "inner" independent variable

$$
\zeta=\frac{z}{\alpha} .
$$

The inner dependent variables are denoted by $\tilde{u}, \tilde{w}, \tilde{p}$ and $\tilde{A}_{i j}$ and the inner problem is now

$$
\begin{aligned}
\epsilon \frac{\alpha^{2}}{\delta} \operatorname{DeRe} D_{t} \tilde{u} & =-\partial_{x} \tilde{p}+\frac{1}{\delta} \operatorname{De}\left(\alpha^{2} \epsilon^{2} \partial_{x}^{2} \tilde{u}+\partial_{\zeta}^{2} \tilde{u}\right)+\epsilon \partial_{x} \tilde{A}_{x x}+\frac{1}{\alpha} \partial_{\zeta} \tilde{A}_{x z}, \\
\epsilon^{3} \frac{\alpha^{2}}{\delta} \operatorname{DeRe} D_{t} \tilde{w} & =-\frac{1}{\alpha} \partial_{\zeta} \tilde{p}+\epsilon^{2} \frac{1}{\delta} \operatorname{De}\left(\alpha^{* 2} \epsilon^{2} \partial_{x}^{2} \tilde{w}+\partial_{\zeta}^{2} \tilde{w}\right)+\epsilon\left(\epsilon \partial_{x} \tilde{A}_{x z}+\frac{1}{\alpha} \partial_{\zeta} \tilde{A}_{z z}\right),
\end{aligned}
$$

$$
\begin{array}{r}
\epsilon \operatorname{De}\left(D_{t} \tilde{A}_{x x}-\frac{2}{\epsilon \alpha} \tilde{A}_{x z} \partial_{\zeta} \tilde{u}-2 \tilde{A}_{x x} \partial_{x} \tilde{u}\right)+\tilde{A}_{x x}-1=\delta\left(\epsilon^{2} \partial_{x}^{2} \tilde{A}_{x x}+\frac{1}{\alpha^{2}} \partial_{\zeta}^{2} \tilde{A}_{x x}\right), \\
\epsilon \operatorname{De}\left(D_{t} \tilde{A}_{x z}-\frac{2}{\epsilon \alpha} \tilde{A}_{z z} \partial_{\zeta} \tilde{u}-\epsilon \tilde{A}_{x x} \partial_{x} w\right)+\tilde{A}_{x z}=\delta\left(\epsilon^{2} \partial_{x}^{2} \tilde{A}_{x z}+\frac{1}{\alpha^{2}} \partial_{\zeta}^{2} \tilde{A}_{x z}\right), \\
\epsilon \operatorname{De}\left(D_{t} \tilde{A}_{z z}-\frac{2}{\alpha} \tilde{A}_{z z} \partial_{\zeta} \tilde{w}-2 \epsilon \tilde{A}_{x z} \partial_{x} \tilde{w}\right)+\tilde{A}_{z z}-1=\delta\left(\epsilon^{2} \partial_{x}^{2} \tilde{A}_{z z}+\frac{1}{\alpha^{2}} \partial_{\zeta}^{2} \tilde{A}_{z z}\right) .
\end{array}
$$

At $\zeta=0$, the boundary conditions are

$$
\tilde{u}=\tilde{w}=0 \quad \text { and } \quad \partial_{\zeta} \tilde{A}_{i j}=0,
$$

with $i=x, \zeta$ and $j=x, \zeta$. We again expand the solution as a regular perturbation series in $\alpha$ :

$$
\begin{gathered}
\tilde{u}=\tilde{u}^{(0)}+\alpha \tilde{u}^{(1)}+\cdots, \quad p=\tilde{p}^{(0)}+\alpha \tilde{p}^{(1)}+\cdots \\
\tilde{w}=\tilde{w}^{(0)}+\alpha \tilde{w}^{(1)}+\cdots, \quad A_{i j}=\tilde{A}_{i j}^{(0)}+\alpha \tilde{A}_{i j}^{(1)}+\cdots
\end{gathered}
$$


To leading order, the inner problem is

$$
\begin{aligned}
& 0=\partial_{\zeta} \tilde{w}^{(0)} \\
& 0=\partial_{\zeta} \tilde{A}_{x z}^{(0)} \\
& 0=-\partial_{\zeta} \tilde{p}^{(0)}+\varepsilon \partial_{\zeta} \tilde{A}_{z z}^{(0)}, \\
& 0=\partial_{\zeta}^{2} \tilde{A}_{x x}^{(0)} \\
& 0=\partial_{\zeta}^{2} \tilde{A}_{x z}^{(0)} \\
& 0=\partial_{\zeta}^{2} \tilde{A}_{z z}^{(0)}
\end{aligned}
$$

with the boundary conditions at $\zeta=0$ :

$$
\tilde{u}^{(0)}=\tilde{w}^{(0)}=0 \quad \text { and } \quad \partial_{\zeta} \tilde{A}_{i j}^{(0)}=0 .
$$

From (4.6a) and $(4.6 \mathrm{~g}), \tilde{w}^{(0)}=0$. From (4.6f) and $(4.6 \mathrm{~g}), \tilde{A}_{i j}^{(0)}$ is a function of $x$ and $t$ only. Hence from (4.6c), $\tilde{p}^{(0)}$ is also independent of $\zeta$.

The next order problem can be written as

$$
\begin{aligned}
& 0=\partial_{x} \tilde{u}^{(0)}+\partial_{\zeta} \tilde{w}^{(1)}, \\
& 0=-\partial_{x} \tilde{p}^{(0)}+\frac{\operatorname{De}}{\delta} \partial_{\zeta}^{2} \tilde{u}^{(0)}+\partial_{\zeta} \tilde{A}_{x z}^{(1)}, \\
& 0=-\partial_{\zeta} \tilde{p}^{(1)}+\varepsilon \partial_{\zeta} \tilde{A}_{z z}^{(1)}, \\
& 0=2 \operatorname{De} \tilde{A}_{x z}^{(0)} \partial_{\zeta} \tilde{u}^{(0)}+\delta \partial_{\zeta}^{2} \tilde{A}_{x x}^{(1)}, \\
& 0=\operatorname{De} \tilde{A}_{z z}^{(0)} \partial_{\zeta} \tilde{u}^{(0)}+\delta \partial_{\zeta}^{2} \tilde{A}_{x z}^{(1)}, \\
& 0=\delta \partial_{\zeta}^{2} \tilde{A}_{z z}^{(1)} .
\end{aligned}
$$

with the boundary conditions at $\zeta=0$

$$
\tilde{u}^{(1)}=\tilde{w}^{(1)}=0 \quad \text { and } \quad \partial_{\zeta} \tilde{A}_{i j}^{(1)}=0 .
$$

From $(4.7 \mathrm{f})$ and $(4.7 \mathrm{~g})$, we see that $\tilde{A}_{z z}^{(1)}$ is a function of $x$ and $t$ only, and from $(4.7 \mathrm{c})$, the same follows for $\tilde{p}^{(1)} ;$ thus

$$
\tilde{A}_{z z}^{(1)}=\tilde{A}_{z z}^{(1)}(x, t), \quad \tilde{p}^{(1)}=\tilde{p}^{(1)}(x, t) .
$$

Differentiation (4.7b) with respect to $\zeta$ and recalling that $\tilde{p}^{(0)}$ is independent of $\zeta$ we have

$$
0=\frac{1}{\delta} \operatorname{De} \partial_{\zeta}^{3} \tilde{u}^{(0)}+\partial_{\zeta}^{2} \tilde{A}_{x z}^{(1)}
$$

using this in (4.7e), we find

$$
0=\tilde{A}_{z z}^{(0)} \partial_{\zeta} \tilde{u}^{(0)}-\partial_{\zeta}^{3} \tilde{u}^{(0)}
$$

Since the $\tilde{A}_{i j}^{(0)}$ are independent of $\zeta$, integrating once gives

$$
\partial_{\zeta}^{2} \tilde{u}^{(0)}-\tilde{A}_{z z}^{(0)} \tilde{u}^{(0)}=c_{1}(x, t)
$$

and for $\tilde{A}_{z z}^{(0)}>0$, we obtain

$$
\tilde{u}^{(0)}=-\frac{c_{1}}{\tilde{A}_{z z}^{(0)}}+c_{2} \exp \left(-\sqrt{\tilde{A}_{z z}^{(0)}} \zeta\right) .
$$


Here we excluded the exponentially growing part, since it does not match to the outer solution. Using the boundary conditions $(4.6 \mathrm{~g})$ yields $c_{2}=c_{1} / \tilde{A}_{z z}^{(0)}$ and hence

$$
\tilde{u}^{(0)}=\frac{c_{1}}{\tilde{A}_{z z}^{(0)}}\left(-1+\exp \left(-\sqrt{\tilde{A}_{z z}^{(0)}} \zeta\right)\right) .
$$

Using this in (4.7b) gives

$$
\begin{aligned}
\tilde{A}_{x z}^{(1)} & =d_{1}(x, t)+\zeta \partial_{x} \tilde{p}^{(0)}-\frac{1}{\delta} \operatorname{De} \partial_{\zeta} \tilde{u}^{(0)} \\
& =d_{1}(x, t)+\zeta \partial_{x} \tilde{p}^{(0)}+\frac{1}{\delta} \operatorname{De} \frac{c_{1}}{\sqrt{\tilde{A}_{z z}^{(0)}}} \exp \left(-\sqrt{\tilde{A}_{z z}^{(0)}} \zeta\right)
\end{aligned}
$$

Due to the last equation in $(4.7 \mathrm{~g})$, we have

$$
\partial_{x} \tilde{p}^{(0)}-\frac{1}{\delta} \operatorname{De} c_{1}=0 \quad \text { or } \quad c_{1}=\delta \frac{\partial_{x} \tilde{p}^{(0)}}{\operatorname{De}},
$$

so that

$$
\begin{aligned}
& \tilde{u}^{(0)}=-\delta \frac{\partial_{x} \tilde{p}^{(0)}}{\operatorname{De} \tilde{A}_{z z}^{(0)}}\left[1-\exp \left(-\sqrt{\tilde{A}_{z z}^{(0)}} \zeta\right)\right], \\
& \tilde{A}_{x z}^{(1)}=d_{1}(x, t)+\zeta \partial_{x} \tilde{p}^{(0)}+\frac{\partial_{x} \tilde{p}^{(0)}}{\sqrt{\tilde{A}_{z z}^{(0)}}} \exp \left(-\sqrt{\tilde{A}_{z z}^{(0)}} \zeta\right) .
\end{aligned}
$$

Notice that $w^{(1)}$ can now be obtained by introducing (4.16a) into (4.7a) and using the boundary condtions $(4.7 \mathrm{~g})$. However, $\tilde{A}_{z z}^{(0)}$ depends on $x$ so the differentiation in (4.7a) creates a rather complicated expression that we do not need in this paper; we omit the explicit result here. For similar reasons, we also skip determining $\tilde{A}_{x x}^{(1)}$, which can in principle be obtained from $(4.7 \mathrm{e}),(4.16)$, and $(4.7 \mathrm{~g})$.

Matching outer solution (at $z=0)$ to the inner $($ as $\zeta \rightarrow \infty)$ yields the following boundary conditions for the leading order outer problem (4.2):

$$
\begin{aligned}
p_{\mid z=0}^{(0)} & =\tilde{p}^{(0)}, \\
A_{i j \mid z=0}^{(0)} & =\tilde{A}_{i j}^{(0)}, \\
u_{\mid z=0}^{(0)} & =-\delta \frac{\partial_{x} p_{\mid z=0}^{(0)}}{\operatorname{De} A_{z z \mid z=0}^{(0)}}, \\
w_{\mid z=0}^{(0)} & =0, \\
\partial_{z} A_{z z \mid z=0}^{(0)} & =0, \\
\partial_{z} A_{x z \mid z=0}^{(0)} & =\partial_{x} p_{\mid z=0}^{(0)} .
\end{aligned}
$$

4.2.1. Inner problem at the free surface. We now consider the inner layer near $z=h$. Introducing inner variables via $z=h-\alpha \zeta$ yields, to leading order in the bulk

$$
\begin{aligned}
\partial_{x} h \partial_{\zeta} \tilde{u}^{(0)}-\partial_{\zeta} \tilde{w}^{(0)} & =0, \\
-\partial_{x} h \partial_{\zeta} \tilde{p}^{(0)}+\varepsilon \partial_{x} h \partial_{\zeta} \tilde{A}_{x x}^{(0)}-\partial_{\zeta} \tilde{A}_{x z}^{(0)} & =0, \\
\partial_{\zeta} \tilde{p}^{(0)}+\varepsilon^{2} \partial_{x} h \partial_{\zeta} \tilde{A}_{x z}^{(0)}-\varepsilon \partial_{\zeta} \tilde{A}_{z z}^{(0)} & =0, \\
\partial_{\zeta}^{2} \tilde{A}_{x x}^{(0)} & =0, \\
\partial_{\zeta}^{2} \tilde{A}_{x z}^{(0)} & =0, \\
\partial_{\zeta}^{2} \tilde{A}_{z z}^{(0)} & =0 .
\end{aligned}
$$


Rescaling and retaining the leading order contributions for the boundary condition at $z=h$ yields

$$
\begin{array}{r}
\partial_{t} h+\tilde{u}^{(0)} \partial_{x} h=\tilde{w}^{(0)} \\
-S_{p} \frac{\partial_{x}^{2} h}{\left[1+\epsilon^{2}\left(\partial_{x} h\right)^{2}\right]^{3 / 2}}=\tilde{p}^{(0)}+\frac{1}{1+\epsilon^{2} h_{x}^{2}}\left\{-\epsilon\left[\tilde{A}_{z z}^{(0)}-1+\epsilon^{2}\left(\partial_{x} h\right)^{2}\left(\tilde{A}_{x x}^{(0)}-1\right)\right]\right. \\
\left.+2 \epsilon^{2} \partial_{x} h \tilde{A}_{x z}^{(0)}\right\} \\
0=\epsilon \partial_{x} h\left(\tilde{A}_{z z}^{(0)}-\tilde{A}_{x x}^{(0)}\right)+\tilde{A}_{x z}^{(0)}\left[1-\epsilon^{2}\left(\partial_{x} h\right)^{2}\right] \\
\partial_{\zeta} \tilde{A}_{i j}^{(0)}=0 .
\end{array}
$$

Integrating (4.18a) with respect to $\zeta$ and using (4.19a) results in

$$
\partial_{t} h+\tilde{u}^{(0)} \partial_{x} h=\tilde{w}^{(0)}
$$

for all $\zeta \geq 0$. the integration constants. Matching this to the outer thus imposes condition (4.19a) onto the leading order outer variables. Integrating (4.18d)-(4.18f) together with (4.19d) shows that the $\tilde{A}_{i j}^{(0)}$ are independent of $\zeta$,

$$
\tilde{A}_{i j}^{(0)}=\tilde{A}_{i j}^{(0)}(x, t) .
$$

Inserting this into (4.18c) gives similarly that $\tilde{p}^{0}$ is independent of $\zeta$

$$
\tilde{p}^{(0)}=\tilde{p}^{(0)}(x, t)
$$

These four functions of $x$ and $t$ need to satisfy the boundary conditions (4.19b) and (4.19c), which matching then passes on to the leading order outer problem. Summarising, we obtain the following conditions at $z=h$ for the leading order outer variables $u^{(0)}, w^{(0)}, A_{i j}^{(0)}$ and $p^{(0)}$ (without the tilde),

$$
\begin{array}{r}
\partial_{t} h+u^{(0)} \partial_{x} h=w^{(0)}, \\
-S_{p} \frac{\partial_{x}^{2} h}{\left[1+\epsilon^{2}\left(\partial_{x} h\right)^{2}\right]^{3 / 2}}=p^{(0)}+\frac{1}{1+\epsilon^{2} h_{x}^{2}}\left\{-\epsilon\left[A_{z z}^{(0)}-1+\epsilon^{2}\left(\partial_{x} h\right)^{2}\left(A_{x x}^{(0)}-1\right)\right]\right. \\
\left.+2 \epsilon^{2} \partial_{x} h A_{x z}^{(0)}\right\} \\
0=\epsilon \partial_{x} h\left(A_{z z}^{(0)}-A_{x x}^{(0)}\right)+A_{x z}^{(0)}\left[1-\epsilon^{2}\left(\partial_{x} h\right)^{2}\right] .
\end{array}
$$

We are now ready to proceed to deriving a thin film flow models.

5. Thin film models. In this section, we derive thin film models for the limit of small film thickness $\epsilon \ll 1$. We consider two cases: one with moderate stress diffusion where $\delta=O(1)$ fixed with respect to $\epsilon$ and $\alpha$, and the other for large stress diffusion $\delta \gg 1$.

5.1. Moderate stress diffusion. We first derive a thin film equation from the sharp interface model; the result shows that singular slip arises in that case. We then re-derive the result from the full model, in order to verify that the slip occurs independent of the order of the limits taken.

5.1.1. Derivation from the sharp interface limit. Taking the limit $\varepsilon \ll 1$ for the sharp-interface model (4.2) together with (4.17c), (4.17e), (4.23a), (4.23b) and (4.23c) yields, to leading order in $\varepsilon$ with 
$\delta=O(1)$ fixed, the problem to solve on $0<z<h$ is

$$
\begin{aligned}
-\partial_{x} p+\partial_{z} A_{x z} & =0, \\
\partial_{z} p & =0, \\
-2 \operatorname{De} A_{x z} \partial_{z} u+A_{x x}-1 & =\delta \partial_{z}^{2} A_{x x}, \\
-\operatorname{De} A_{z z} \partial_{z} u+A_{x z} & =\delta \partial_{z}^{2} A_{x z}, \\
A_{z z}-1 & =\delta \partial_{z}^{2} A_{z z}, \\
\partial_{x} u+\partial_{z} w & =0 .
\end{aligned}
$$

We apply the following at $z=0$ :

Note that (5.11) arises from matching and it is derived in the appendix. We have dropped the superscript "(0)" from the variables for convenience.

Using $(5.1 \mathrm{~b})$ and $(5.1 \mathrm{j})$ gives

$$
p=p(x, t)=-S_{p} \partial_{x}^{2} h .
$$

From (5.1a) we have

$$
\partial_{z} A_{x z}=-S_{p} \partial_{x}^{3} h,
$$

and from $(5.1 \mathrm{k})$

$$
A_{x z}=S_{p}(h-z) \partial_{x}^{3} h .
$$

Integrating (5.1e) and using (5.1h) and (5.11) results in

$$
A_{z z}=1 .
$$

Then from (5.1d),

$$
\text { De } \partial_{z} u=-\delta \partial_{z}^{2} A_{x z}+A_{x z}=S_{p}(h-z) \partial_{x}^{3} h .
$$

Using (5.1g), (5.2) and $A_{z z}=1$ gives the following boundary condition at $z=0$ :

$$
u=\frac{\delta S_{p}}{\operatorname{De}} \partial_{x}^{3} h .
$$

From this and (5.6),

$$
u=\frac{S_{p}}{\operatorname{De}} \partial_{x}^{3} h\left(z h-\frac{z^{2}}{2}+\delta\right) .
$$

If we assume $\left.w\right|_{z=0}=0$ then

$$
\partial_{t} h+\partial_{x} \int_{0}^{h} u d z=0
$$


holds and using (5.8), we obtain

$$
\partial_{t} h+\partial_{x}\left[\frac{S_{p}}{\mathrm{De}} \partial_{x}^{3} h\left(\frac{h^{3}}{3}+\delta h\right)\right]=0
$$

This is a lubrication model with an $h$-dependent singular slip-length

$$
b=\frac{\delta}{h}
$$

used by Greenspan [12] and derived in Huh \& Scriven [13] and in Neogi \& Miller [23]. This result illustrates how the apparent slip appears in the context of the nonlinear thin film equation, and that it is because there is a boundary layer in the velocity profile inside the film. However, one may ask whether this is because $\alpha \ll 1$ was taken prior to deriving the thin film equation. We address this point by deriving the same result from the full equations and taking the limit $\alpha \ll 1$ after deriving a thin film equation.

5.1.2. Derivation from the full governing equations. We now directly derive a thin film model for the governing equations, where $\alpha$ is treated as a fixed constant and $\epsilon \ll 1$.

For conservation of mass, we have

$$
\partial_{x} u+\partial_{z} w=0 .
$$

For momentum conservation,

$$
\begin{aligned}
& 0=-\partial_{x} p+\frac{\alpha^{2}}{\delta} \operatorname{De} \partial_{z}^{2} u+\partial_{z} A_{x z}, \\
& 0=-\partial_{z} p .
\end{aligned}
$$

For the polymer part of the deviatoric stress, the equations are as follows.

$$
\begin{aligned}
-2 \operatorname{De} A_{x z} \partial_{z} u+A_{x x}-1 & =\delta \partial_{z}^{2} A_{x x}, \\
-\operatorname{De} A_{z z} \partial_{z} u+A_{x z} & =\delta \partial_{z}^{2} A_{x z}, \\
A_{z z}-1 & =\delta \partial_{z}^{2} A_{z z} .
\end{aligned}
$$

Note that our choice of large pressure makes it larger than the leading order $A_{z z}$ term.

The boundary conditions are, at $z=0$,

$$
u=w=0 \text { and } \partial_{z} A_{i j}=0,
$$

with $i=x, z$ and $j=x, z$.

On the free surface $z=h$,

$$
\begin{aligned}
\partial_{t} h-u \partial_{x} h & =w, \\
-S_{p} \frac{\partial_{x}^{2} h}{\left[1+\epsilon^{2}\left(\partial_{x} h\right)^{2}\right]^{3 / 2}} & =p, \\
A_{x z}+\frac{\alpha^{2}}{\delta} \operatorname{De} \partial_{z} u & =0, \\
\partial_{z} A_{i j} & =0 .
\end{aligned}
$$

To solve this system, first consider $A_{z z}$ since it is a linear equation. Applying the homogeneous Neumann boundary conditions $(5.12 \mathrm{~g})$ and $(5.12 \mathrm{k})$ yields

$$
A_{z z}=1,
$$

i.e. the polymer stress state is uniform across the thin film. The polymer stress state for the other components will not be uniform in $z$. We integrate the momentum equation (5.12b) and use the tangential stress boundary condition $(5.12 \mathrm{k})$ as well as (5.13) to obtain

$$
\frac{\alpha^{2}}{\delta} \operatorname{De} \partial_{z} u=-A_{x z}+(z-h) \partial_{x} p .
$$


Now substituting for $A_{z z}$ and $\partial_{z} u$ in the polymer shear stress equation gives

$$
\frac{\alpha^{2}}{1+\alpha^{2} / \delta} \partial_{z}^{2} A_{x z}-A_{x z}=-\frac{1}{1+\alpha^{2} / \delta}(z-h) \partial_{x} p .
$$

The solution which satisfies the boundary conditions $(5.12 \mathrm{~g})$ and $(5.12 \mathrm{k})$ is

$$
A_{x z}=\frac{\partial_{x} p}{1+\alpha^{2} / \delta}\left\{z-h+\alpha \frac{\cosh \left(\frac{\sqrt{1+\alpha^{2} / \delta}}{\alpha}(z-h)\right)-\cosh \left(\frac{\sqrt{1+\alpha^{2} / \delta}}{\alpha} z\right)}{\sqrt{1+\alpha^{2} / \delta} \sinh \left(\frac{\sqrt{1+\alpha^{2} / \delta}}{\alpha} h\right)}\right\} .
$$

Since $\partial_{z} p=0$ in the film, then the normal stress boundary conditions determines that

$$
\partial_{x} p=-S_{p} \partial_{x}^{3} h
$$

The solution for $u$ is then

$$
\begin{aligned}
u= & \frac{1}{\left(1+\alpha^{2} / \delta\right)} \frac{S_{p}}{\operatorname{De}} \partial_{x}^{3} h\left\{-\left(\frac{z^{2}}{2}-z h\right)\right. \\
& \left.+\frac{\delta}{1+\alpha^{2} / \delta}\left[1+\frac{\sinh \left(\frac{\sqrt{1+\alpha^{2} / \delta}}{\alpha}(z-h)\right)-\sinh \left(\frac{\sqrt{1+\alpha^{2} / \delta}}{\alpha} z\right)}{\sinh \left(\frac{\sqrt{1+\alpha^{2} / \delta}}{\alpha} h\right)}\right]\right\} .
\end{aligned}
$$

The evolution of the free boundary $h(x, t)$ is given by

$$
\partial_{t} h+\partial_{x} q=0, \quad q=\int_{0}^{h} u d z
$$

with

$$
q=\frac{1}{1+\alpha^{2} / \delta} \frac{S_{p}}{\operatorname{De}}\left\{\frac{h^{3}}{3}+\frac{\delta h}{1+\alpha^{2} / \delta}\left[1+\frac{2 \alpha\left(1-\cosh \left(\frac{\sqrt{1+\alpha^{2} / \delta}}{\alpha} h\right)\right)}{\sqrt{1+\alpha^{2} / \delta} h \sinh \left(\frac{\sqrt{1+\alpha^{2} / \delta}}{\alpha} h\right)}\right]\right\} \partial_{x}^{3} h
$$

This complicated equation for the flux is the integral of $u$ across the film where $u$ has the potential to develop boundary layers if $\alpha$ is small enough. When $\alpha$ is not vanishingly small, then the flow near the boundaries still contributes significantly to the flux.

If we now take the sharp interface limit $\alpha \rightarrow 0$ in (5.20), we recover the previously obtained thin film model (5.10). In this case, the flow away from the boundaries gives the dominant contribution to the flux $q$ inside the film due to the vanishing width of the boundary layers. Thus, for $\delta=O(1)$ fixed, the order of the limits $\alpha \ll 1$ and $\epsilon \ll 1$ is immaterial regarding whether slip arises in the resulting thin film equations.

5.2. Large stress diffusion. For the materials considered in this study, the typical parameter regimes are covered by the above asymptotic cases, see Table 1 . However, further asymptotic regimes that account for large slip are possible. One of these cases, with large diffusion, is treated now. In this case we see from (5.12b) that $u \sim \delta$. We therefore rescale

$$
(u, w)=\delta(\bar{u}, \bar{w}), \quad t=\frac{\bar{t}}{\delta},
$$

but keep the same scaling for $A_{x z}$ and $A_{z z}$.

For conservation of mass we then have

$$
\partial_{x} u+\partial_{z} w=0 .
$$


For momentum conservation,

$$
\begin{aligned}
\epsilon \alpha^{2} \delta \operatorname{DeRe} D_{t} u & =-\partial_{x} p+\alpha^{2} \operatorname{De}\left(\epsilon^{2} \partial_{x}^{2} u+\partial_{z}^{2} u\right)+\epsilon \partial_{x} A_{x x}+\partial_{z} A_{x z}, \\
\epsilon^{3} \alpha^{2} \delta \operatorname{DeRe} D_{t} w & =-\partial_{z} p+\epsilon^{2} \alpha^{2} \operatorname{De}\left(\epsilon^{2} \partial_{x}^{2} w+\partial_{z}^{2} w\right)+\epsilon\left(\epsilon \partial_{x} A_{x z}+\partial_{z} A_{z z}\right) .
\end{aligned}
$$

For the polymer part of the deviatoric stress, the equations are as follows.

$$
\begin{array}{r}
\epsilon \delta \operatorname{De}\left(D_{t} A_{x x}-2 \epsilon^{-1} A_{x z} \partial_{z} u-2 \epsilon A_{x x} \partial_{x} u\right)+A_{x x}-1=\delta\left(\epsilon^{2} \partial_{x}^{2} A_{x x}+\partial_{z}^{2} A_{x x}\right), \\
\epsilon \operatorname{De} \delta\left(D_{t} A_{x z}-\epsilon^{-1} A_{z z} \partial_{z} u-\epsilon A_{x x} \partial_{x} w\right)+A_{x z}=\delta\left(\epsilon^{2} \partial_{x}^{2} A_{x z}+\partial_{z}^{2} A_{x z}\right), \\
\epsilon \operatorname{De} \delta\left(D_{t} A_{z z}-2 A_{z z} \partial_{z} w-2 \epsilon A_{x z} \partial_{x} w\right)+A_{z z}-1=\delta\left(\epsilon^{2} \partial_{x}^{2} A_{z z}+\partial_{z}^{2} A_{z z}\right) .
\end{array}
$$

Note that our choice of large pressure i.e. of the scaling $G_{0} / \varepsilon$ for $p^{\prime}$ in (2.2) makes it larger than the leading order $A_{z z}$ term.

The boundary conditions are, at $z=0$,

$$
u=w=0 \quad \text { and } \quad \partial_{z} A_{i j}=0,
$$

with $i=x, z$ and $j=x, z$.

We now turn to the free surface boundary conditions at $z=h(x, t)$. We have

$$
\mathbf{n}=\left(-\epsilon \partial_{x} h \mathbf{i}+\mathbf{j}\right) N^{-1}, \quad \mathbf{t}=\left(\mathbf{i}+\epsilon \partial_{x} h \mathbf{j}\right) N^{-1}, \quad \text { and } \quad N=\sqrt{1+\left(\epsilon \partial_{x} h\right)^{2}} .
$$

On the free surface $z=h$,

$$
\partial_{t} h-u \partial_{x} h=w
$$

$$
\begin{aligned}
-S_{p} \frac{\partial_{x}^{2} h}{\left[1+\epsilon^{2}\left(\partial_{x} h\right)^{2}\right]^{3 / 2}=} & p+\frac{1}{1+\epsilon^{2} h_{x}^{2}}\left\{-\epsilon\left[A_{z z}-1+\epsilon^{2}\left(\partial_{x} h\right)^{2}\left(A_{x x}-1\right)\right]\right. \\
& -2 \alpha^{2} \operatorname{De}^{2} \partial_{x} h\left(\partial_{z} u+\epsilon^{2} \partial_{x} w\right) \\
& \left.-2 \epsilon \partial_{x} h A_{x z}+2 \alpha^{2} \operatorname{De}^{2}\left[\partial_{z} w+\epsilon^{2}\left(\partial_{x} h\right)^{2} \partial_{x} u\right]\right\} ;
\end{aligned}
$$

$$
\begin{aligned}
& 0=\epsilon \partial_{x} h\left(A_{z z}-A_{x x}\right)+A_{x z}\left[1-\epsilon^{2}\left(\partial_{x} h\right)^{2}\right] \\
& +\alpha^{2} \operatorname{De}\left(\partial_{z} u+\epsilon^{2} \partial_{x} w\right)\left[1-\epsilon^{2}\left(\partial_{x} h\right)^{2}\right]-2 \alpha^{2} \operatorname{De} \epsilon \partial_{x} h\left(\partial_{x} u-\partial_{z} w\right) ;
\end{aligned}
$$

and

$$
\left(\partial_{z} A_{i j}-\epsilon^{2} \partial_{x} h \partial_{x} A_{i j}\right) N^{-1}=0 .
$$

5.2.1. Leading order equations. We consider the $O(1)$ problem for $\varepsilon \ll 1$ and $\delta \gg 1$, keeping $\alpha$ fixed. For conservation of mass we have

$$
\partial_{x} u+\partial_{z} w=0
$$

For momentum conservation,

$$
\begin{aligned}
& 0=-\partial_{x} p+\alpha^{2} \operatorname{De} \partial_{z}^{2} u+\partial_{z} A_{x z}, \\
& 0=\partial_{z} p
\end{aligned}
$$

where, for the sake of simplicity, we have also assumed that DeRe $\ll 1$. Using the definitions of De and Re, this requires $U \ll \sqrt{\eta_{s} / \rho / \lambda} \approx 10^{-3} \mathrm{~m} / \mathrm{s}$; from the values in Table 1 we see that this is readily achieved.

For the polymer part of the deviatoric stress, the equations are as follows.

$$
\begin{aligned}
-2 \operatorname{De} A_{x z} \partial_{z} u & =\partial_{z}^{2} A_{x x}, \\
-\operatorname{De} A_{z z} \partial_{z} u & =\partial_{z}^{2} A_{x z}, \\
0 & =\partial_{z}^{2} A_{z z} .
\end{aligned}
$$


He we see the consequence of our choice that the pressure is larger than the leading order $A_{z z}$ term.

The boundary conditions are, at $z=0$,

$$
u=w=0 \text { and } \partial_{z} A_{i j}=0,
$$

with $i=x, z$ and $j=x, z$.

On the free surface $z=h$,

and

$$
\begin{gathered}
\partial_{t} h-u \partial_{x} h=w ; \\
-S_{p} \partial_{x}^{2} h=p \\
0=A_{x z}+\alpha^{2} \operatorname{De} \partial_{z} u .
\end{gathered}
$$

The solution for $A_{z z}$ is

$$
A_{z z}=A_{1}(x, t)
$$

with an unknown (but $z$-independent) function $A_{1}$. Furthermore,

$$
\begin{gathered}
u=D_{1}(x, t) \sinh \left(z \frac{\sqrt{A_{1}(x, t)}}{\alpha}\right)+\frac{\partial_{x} p}{\operatorname{De} A_{1}(x, t)}\left[\cosh \left(z \frac{\sqrt{A_{1}(x, t)}}{\alpha}\right)-1\right], \\
A_{x z}=-\alpha \operatorname{De} \sqrt{A_{1}(x, t)} D_{1}(x, t) \cosh \left(z \frac{\sqrt{A_{1}(x, t)}}{\alpha}\right) \\
+\partial_{x} p\left[\frac{-\alpha}{\sqrt{A_{1}(x, t)}} \sinh \left(z \frac{\sqrt{A_{1}(x, t)}}{\alpha}\right)+z-h\right] .
\end{gathered}
$$

$D_{1}$ can be eliminated from these solutions by using (5.33), resulting in

$$
D_{1}(x, t)=\frac{\partial_{x} p}{\operatorname{De} A_{1}(x, t)} \frac{1-\cosh \left(h \frac{\sqrt{A_{1}(x, t)}}{\alpha}\right)}{\sinh \left(h \frac{\sqrt{A_{1}(x, t)}}{\alpha}\right)} .
$$

Letting $\alpha \rightarrow 0$ we find

$$
D_{1} \rightarrow-\frac{\partial_{x} p}{\operatorname{De} A_{1}(x, t)} .
$$

Using this in (5.35) yields (in the same limit)

$$
u(x, t)=-\frac{\partial_{x} p}{\operatorname{De} A_{1}(x, t)} .
$$

5.2.2. Next order problem: Distinguished limit $\mathbf{1} / \boldsymbol{\delta}=\boldsymbol{d} \boldsymbol{\epsilon}$. To determine $A_{1}(x, t)$, we need to consider the next order problem. For this purpose we assume the distinguished limit $1 / \delta=d \epsilon$ with

$$
d=\frac{1}{\delta \epsilon}=O(1) .
$$

For simplicity let Re $\ll 1$ be negligible. In the bulk, we only need to consider (5.22f), which becomes

$$
\operatorname{De}\left(\epsilon D_{t} A_{z z}-2 \epsilon A_{z z} \partial_{z} w-2 \epsilon^{2} A_{x z} \partial_{x} w\right)+d \epsilon\left(A_{z z}-1\right)=\epsilon^{2} \partial_{x}^{2} A_{z z}+\partial_{z}^{2} A_{z z} .
$$


Its boundary conditions are, at $z=0$,

$$
\partial_{z} A_{z z}=0
$$

and at $z=h(x, t)$,

$$
\left(\partial_{z} A_{i j}-\epsilon^{2} \partial_{x} h \partial_{x} A_{i j}\right) N^{-1}=0 .
$$

Now expand with a regular series in powers of $\epsilon$,

$$
A_{z z}=A_{z z}^{(0)}+\epsilon A_{z z}^{(1)}+\ldots,
$$

and similarly for the other variables. Leading order is as for the moderate $\delta$ case (simply insert $(0)$ superscripts). To next order we obtain, for the polymer stress equation,

$$
\operatorname{De}\left(D_{t} A_{z z}^{(0)}-2 A_{z z}^{(0)} \partial_{z} w^{(0)}\right)+d\left(A_{z z}^{(0)}-1\right),=\partial_{z}^{2} A_{z z}^{(1)}
$$

and for the boundary conditions at $z=0$ and $z=h(x, t)$,

$$
\partial_{z} A_{z z}^{(1)}=0 .
$$

We then substitute the solution from (5.34) into (5.43) and integrate with respect to $z$ from 0 to $h(x, t)$; using the boundary condtions yields the solvabilitiy condition

$$
\partial_{t} A_{1}+u \partial_{x} A_{1}+2 A_{1} \partial_{x} u+\frac{d}{\operatorname{De}}\left(A_{1}-1\right)=0
$$

where we have omitted the superscripts from $u$. Note that $u$ from (5.39) is independent of $z$ so that mass conservation yields

$$
\partial h+\partial_{x}(u h)=0 .
$$

Combining equations $(5.31),(5.39),(5.45),(5.46)$ gives the system

$$
\begin{aligned}
\partial_{t} A_{1}+\frac{S_{p}}{\operatorname{De}}\left[2 \partial_{x}^{4} h-\partial_{x}^{3} h \frac{\partial_{x} A_{1}}{A_{1}}\right]+\frac{d}{\operatorname{De}}\left(A_{1}-1\right) & =0, \\
\partial_{t} h+\frac{S_{p}}{\operatorname{De}} \partial_{x}\left(\frac{h \partial_{x}^{3} h}{A_{1}}\right) & =0 .
\end{aligned}
$$

We note that in the limit $d \gg 1$, we obtain $A_{1} \equiv 1$ and thus get from $(5.47 \mathrm{~b})$

$$
\partial_{t} h+\frac{S_{p}}{\operatorname{De}} \partial_{x}\left(h \partial_{x}^{3} h\right)=0
$$

This is the same evolution equation that results from the $\delta \rightarrow \infty$ limit of (5.10) after rescaling time according to (5.21). We now turn to solving a simple example problem from this system.

5.2.3. Linear stability of the uniform solution. To gain some insight into the large stress diffusion model, we consider sinusoidal disturbances to the temporally- and spatially-uniform solutions $h=A_{1}=1$ on an infinite domain. We find that these uniform states are stable, so this is analogous to the leveling problem [25]. We may write

$$
h(x, t)=1+\tilde{\epsilon} \mathcal{H}(t) e^{i k x} \text { and } A_{1}(x, t)=1+\tilde{\epsilon} \mathcal{A}(t) e^{i k x},
$$

where $\tilde{\epsilon} \ll 1$ and the amplitudes $\mathcal{H}$ and $\mathcal{A}$ may be complex valued (we omitted the complex conjugate term for simplicity). The initial values of the amplitudes are $\mathcal{A}(0)=\mathcal{A}_{0}$, and $\mathcal{H}(0)=\mathcal{H}_{0}$. Substitution into equations (5.47a) and (5.47b), keeping terms of $O(\tilde{\epsilon})$ only, results in the following linear system of ODEs for the amplitude:

$$
\begin{aligned}
\dot{\mathcal{A}}+\frac{2 S_{p} k^{4}}{\mathrm{De}} \mathcal{H}+\frac{d}{\mathrm{De}} \mathcal{A} & =0, \\
\dot{\mathcal{H}}+\frac{S_{p} k^{4}}{\mathrm{De}} \mathcal{H} & =0 .
\end{aligned}
$$


The dots denote time derivatives. Solving this system gives

$$
\begin{aligned}
& \mathcal{A}(t)=\left(\mathcal{A}_{0}-\mathcal{H}_{0} \frac{2 S_{p} k^{4}}{S_{p} k^{4}-d}\right) \exp \left(-\frac{d}{\mathrm{De}} t\right)+\mathcal{H}_{0} \frac{2 S_{p} k^{4}}{S_{p} k^{4}-d} \exp \left(-\frac{S_{p} k^{4}}{\mathrm{De}} t\right), \\
& \mathcal{H}(t)=\mathcal{H}_{0} \exp \left(-\frac{S_{p} k^{4}}{\mathrm{De}} t\right) .
\end{aligned}
$$

Note that the terms proportional to $\mathcal{H}_{0}$ that appear in 5.50a are in phase provided that $S_{p} k^{4}-d>0$, that is, for sufficiently short waves; otherwise they are out of phase with the wavenumber-independent polymer stress decay rate.

There are two time scales for decay for this linearized problem. One is $d / D e$ from internal polymer stress relaxation, and the other is $S_{p} k^{4}$ De which is from surface tension. The polymer stress relaxation scale is faster if $k<k_{c}=\left(d / S_{p}\right)^{1 / 4}$. Using the values from Table 1, for CTAB 25/25 and for CPyCl/NaSal 50/25, we have $k_{c} \sim 100$, so that for any long wave situation the polymer stress relaxation will be faster than the capillarity-driven decay. If we use $H=10^{-5} \mathrm{~m}$ and $\ell=10^{-3} \mathrm{~m}$, then $k_{c} \sim 10$, and a similar conclusion may be drawn. For $k=1, d /$ De $\gg S_{p} /$ De and the time scales differ by about seven orders of magnitude for $\mathrm{CPyCl} / \mathrm{NaSal} 50 / 25$; the scales differ by orders of magnitude for all materials in Table 1.

6. Discussion and Outlook. In this paper we have considered planar channel flow and thin film free surface flows governed by a diffusive upper convected Maxwell model of a micelle solution with a Newtonian solvent. For a pressure driven channel flow the flow structure and dynamics, namely the formation of boundary layers and the transitions in the flow field, are controlled by two parameters: The ratio of the solvent viscosity to the zero shear rate polymer viscosity $\eta_{s} / \eta_{0}=1 /\left(1+\eta_{0}^{p} / \eta_{s}\right)$, and the non-dimensional stress diffusion parameter $\delta$. Since usually $\eta_{s} / \eta_{0}^{p} \ll 1$, this ratio can also be considered instead of the former. We have shown that this viscosity ratio together with $\delta$ control the thickness of the boundary layer $\alpha$, while the magnitude of $\delta$ determines the magnitude of the apparent slip.

This connection can be rationalized by treating the channel flow (or the thin film flow) as a two-layer flow, with a bulk flow near the center (or near the free surface, respectively) and thin layer of width $\alpha$ at the boundaries (or the substrate).

As shown in a study of bi-layer thin film models by Jachalski et al. [14], the flow in a layer of viscosity $\eta_{2}$ on top of another layer of height $h_{1}$ and much smaller viscosity $\eta_{1}$ adjacent to a substrate experiences an apparent slip of $h_{1} /\left(\eta_{1} / \eta_{2}\right)$.

In fact, effective viscosities $\eta_{\text {eff }}^{\text {center }}$ and $\eta_{\text {eff }}^{\text {layer }}$ can be obtained for each of these regions from the ratio $A_{x z}^{\prime} / \partial_{z^{\prime}} u^{\prime}$ as $z^{\prime} \rightarrow 0$ and $z^{\prime} \rightarrow-H / 2$,

$$
\eta_{\mathrm{eff}}^{\text {center }}=\lim _{z^{\prime} \rightarrow 0} \frac{A_{x z}^{\prime}}{\partial_{z^{\prime}} u^{\prime}}=\eta_{p}^{0}, \quad \eta_{\mathrm{eff}}^{\text {layer }}=\lim _{z^{\prime} \rightarrow-H / 2} \frac{A_{x z}^{\prime}}{\partial_{z^{\prime}} u^{\prime}}=\eta_{p}^{0} \frac{\alpha}{2 \delta},
$$

where the limits have been evaluated by using (3.2) and (3.3) together with the scalings and definitions (2.2) and (2.3), assuming that $\delta$ is fixed and $\alpha \ll 1$ (which follows from $\eta_{p}^{0} \gg \eta_{s}$ and $\delta$ fixed). Thus, using $\eta_{\mathrm{eff}}^{\text {layer }}$ for $\eta_{1}$ and $\eta_{\text {eff }}^{\text {center }}$ for $\eta_{2}$, and $h_{1}=\alpha$, we obtain the apparent slip-length for the outer channel flow

$$
\frac{\alpha}{\eta_{\text {eff }}^{\text {layer }} / \eta_{\text {eff }}^{\text {center }}}=2 \delta
$$

i.e. the same value we obtained previously from (3.6) and the $\alpha \rightarrow 0$ limit of (3.3).

The limit $\alpha \rightarrow 0$ was also considered for thin films with a free capillary surface and thin-film models were derived both for the case of moderate ( $\delta$ fixed) and large $\left(\delta=O\left(\varepsilon^{-1}\right)\right)$ stress diffusion. Interestingly, these models show several parallels with those derived earlier in the context of a liquid layer of polymer melt dewetting from hydrophobized substrate using a Navier-slip condition for slip-lengths of various orders of magnitude; see Münch et al. [20, 22] and Fetzer et al. [10].

Here, the distinction between the two cases is similar to what was found for thin-film models with weak and strong slip in [22], confirming the association of slip with the parameter $\delta$ also in the case of thin film flows. Due to the choice of the regime for the Deborah number De, the models correspond to those expected for a Newtonian rheology in the bulk. In contrast to [22], however, the models here correspond to slip laws with a slip length that has a singular dependence on the film profile $h$. 
Our analysis of the sharp-interface limit $\alpha \rightarrow 0$ and the derivation of several thin-film models for the simplest type of model of micelle solutions suggest further investigations into other regimes of Deborah number, in particular in the case of large stress diffusion, where corresponding strong-slip type thin film models even for full nonlinear viscoelastic rheologies [21] can be expected. Further work will consider extensions of our stability analysis for these models together with numerical solutions of the thin-film models.

\section{Appendix A. Matching to the inner solution at $\mathrm{z}=\mathbf{h}$.}

We now determine $A_{z z}$, which is in fact the leading order approximation of the outer solution, i.e. $A_{z z}^{(0)}$. Matching also requires the next order correction to the inner problem near $z=h$. In terms of the inner variables near $z=h$,

$$
\zeta=\frac{h(x, t)-z}{\alpha}
$$

we obtain to next order in $\alpha$ the problem

At the free surface, $\zeta=0$,

$$
\partial_{\zeta}^{2} \tilde{A}_{x x}^{(1)}=\frac{\operatorname{De}}{\delta} \frac{2}{1+\varepsilon^{2}\left(\partial_{x} h\right)^{2}}\left(\tilde{A}_{x z}^{(0)}-\varepsilon \partial_{x} h \tilde{A}_{x x}^{(0)}\right) \partial_{\zeta} \tilde{u}^{(0)},
$$

$$
\begin{aligned}
& \partial_{\zeta}^{2} \tilde{A}_{x z}^{(1)}=\frac{\mathrm{De}}{\delta} \frac{1}{1+\varepsilon^{2}\left(\partial_{x} h\right)^{2}}\left(\tilde{A}_{z z}^{(0)} \partial_{\zeta} \tilde{u}^{(0)}-\varepsilon^{2} \partial_{x} h \tilde{A}_{x x}^{(0)} \partial_{\zeta} \tilde{w}^{(0)}\right), \\
& \partial_{\zeta}^{2} \tilde{A}_{z z}^{(1)}=\frac{\mathrm{De}}{\delta} \frac{2 \varepsilon}{1+\varepsilon^{2}\left(\partial_{x} h\right)^{2}}\left(\tilde{A}_{z z}^{(0)}-\varepsilon \partial_{x} h \tilde{A}_{x z}^{(0)}\right) \partial_{\zeta} \tilde{w}^{(0)} .
\end{aligned}
$$

$$
\begin{aligned}
\partial_{x} h \tilde{u}^{(1)}= & \tilde{w}^{(1)}, \\
0= & \tilde{p}^{(1)}+\frac{\mathrm{De}}{\delta} 2 \varepsilon^{2}\left(\partial_{x} h \partial_{\zeta} \tilde{u}^{(0)}-\partial_{\zeta} \tilde{w}^{(0)}\right) \\
& +\frac{\varepsilon}{1+\varepsilon^{2}\left(\partial_{x} h\right)^{2}}\left(\varepsilon\left(\partial_{x} h\right)^{2} \tilde{A}_{x x}^{(1)}+2 \varepsilon \partial_{x} h \tilde{A}_{x z}^{(1)}-\tilde{A}_{z z}^{(1)}\right), \\
0= & \varepsilon \partial_{x} h\left(\tilde{A}_{z z}^{(1)}-\tilde{A}_{x x}^{(1)}\right) \\
& +\left[\tilde{A}_{x z}^{(1)}+\frac{\mathrm{De}}{\delta}\left(-\partial_{\zeta} \tilde{u}^{(0)}+\varepsilon^{2} \partial_{x} h \partial_{\zeta} \tilde{w}^{(0)}\right)\right]\left(1-\varepsilon^{2}\left(\partial_{x} h\right)^{2}\right) \\
& -\frac{\mathrm{De}}{\delta} 2 \varepsilon \partial_{x} h\left(\partial_{x} h \partial_{\zeta} \tilde{u}^{(0)}+\partial_{\zeta} \tilde{w}^{(0)}\right), \\
\partial_{\zeta} \tilde{A}_{i j}^{(1)}= & -\frac{\varepsilon^{3} \partial_{x} h}{1+\varepsilon^{2}\left(\partial_{x} h\right)^{2}} \partial_{x} \tilde{A}_{i j}^{(0)} .
\end{aligned}
$$

This problem can be simplified by introducing the new variables

$$
\tilde{q}^{(0)}=\partial_{x} h \tilde{u}^{(0)}-\tilde{w}^{(0)} \quad \text { and } \quad \tilde{r}^{(0)}=\tilde{u}^{(0)}+\varepsilon^{2} \partial_{x} h \tilde{w}^{(0)} .
$$


643

644

645

646

647

648

649

650

651

652

653

654

655

656

657

658

659

660

661

662

663

664

665

666

667

668

669

670

671

672

673

instead of $\tilde{u}^{(0)}$ and $\tilde{w}^{(0)}$. Using these variables, we obtain

(A.3a)

$$
\begin{aligned}
& 0=\partial_{\zeta} \tilde{q}^{(0)} \\
& 0=-\partial_{x} \tilde{p}^{(0)}-\partial_{x} h \partial_{\zeta} \tilde{p}^{(1)}+\frac{\mathrm{De}}{\delta} \partial_{\zeta}^{2} \tilde{r}^{(0)}-\partial_{\zeta} \tilde{A}_{x z}^{(1)}
\end{aligned}
$$$$
+\varepsilon \partial_{x} \tilde{A}_{x x}^{(0)}+\varepsilon \partial_{x} h \partial_{\zeta} \tilde{A}_{x x}^{(1)} \text {, }
$$$$
0=\partial_{\zeta} \tilde{p}^{(1)}+\frac{\operatorname{De}}{\delta} \frac{1+\varepsilon^{2}\left(\partial_{x} h\right)^{2}}{\partial_{x} h} \partial_{\zeta}^{2} \tilde{r}^{(0)}-\varepsilon \partial_{\zeta} \tilde{A}_{z z}^{(1)}
$$

$$
+\varepsilon^{2} \partial_{x} \tilde{A}_{x z}^{(0)}+\varepsilon^{2} \partial_{x} h \partial_{\zeta} \tilde{A}_{x z}^{(1)},
$$

$$
\partial_{\zeta}^{2} \tilde{A}_{x x}^{(1)}=\frac{\mathrm{De}}{\delta} \frac{2}{\left(1+\varepsilon^{2}\left(\partial_{x} h\right)^{2}\right)^{2}}\left(\tilde{A}_{x z}^{(0)}-\varepsilon \partial_{x} h \tilde{A}_{x x}^{(0)}\right) \partial_{\zeta} \tilde{r}^{(0)},
$$

$$
\begin{aligned}
& \partial_{\zeta}^{2} \tilde{A}_{x z}^{(1)}=\frac{\mathrm{De}}{\delta} \frac{1}{\left(1+\varepsilon^{2}\left(\partial_{x} h\right)^{2}\right)^{2}}\left(\tilde{A}_{z z}^{(0)}-\varepsilon^{2}\left(\partial_{x} h\right)^{2} \tilde{A}_{x x}^{(0)}\right) \partial_{\zeta} \tilde{r}^{(0)}, \\
& \partial_{\zeta}^{2} \tilde{A}_{z z}^{(1)}=\frac{\mathrm{De}}{\delta} \frac{2 \varepsilon \partial_{x} h}{\left(1+\varepsilon^{2}\left(\partial_{x} h\right)^{2}\right)^{2}}\left(\tilde{A}_{z z}^{(0)}-\varepsilon \partial_{x} h \tilde{A}_{x z}^{(0)}\right) \partial_{\zeta} \tilde{r}^{(0)} .
\end{aligned}
$$

The boundary conditions at $\zeta=0$ become:

$$
\partial_{x} h \tilde{u}^{(1)}=\tilde{w}^{(1)},
$$$$
0=\tilde{p}^{(1)}+\frac{\varepsilon}{1+\varepsilon^{2}\left(\partial_{x} h\right)^{2}}\left(\varepsilon\left(\partial_{x} h\right)^{2} \tilde{A}_{x x}^{(1)}+2 \varepsilon \partial_{x} h \tilde{A}_{x z}^{(1)}-\tilde{A}_{z z}^{(1)}\right),
$$

$$
\begin{aligned}
0= & \varepsilon \partial_{x} h\left(\tilde{A}_{z z}^{(1)}-\tilde{A}_{x x}^{(1)}\right)+\tilde{A}_{x z}^{(1)}\left(1-\varepsilon^{2}\left(\partial_{x} h\right)^{2}\right) \\
& -\frac{\operatorname{De}}{\delta} \frac{\left[\left(1-\varepsilon^{2}\left(\partial_{x} h\right)^{2}\right)^{2}+4 \varepsilon\left(\partial_{x} h\right)^{2}\right]}{1+\varepsilon^{2}\left(\partial_{x} h\right)^{2}} \partial_{\zeta} \tilde{r}^{(0)},
\end{aligned}
$$

$$
\partial_{\zeta} \tilde{A}_{i j}^{(1)}=-\frac{\varepsilon^{3} \partial_{x} h}{1+\varepsilon^{2}\left(\partial_{x} h\right)^{2}} \partial_{x} \tilde{A}_{i j}^{(0)} .
$$

Adding (A.3b) to (A.3c) multiplied by $\partial_{x} h$, we obtain

$$
\begin{aligned}
\frac{\mathrm{De}}{\delta}\left[2+\varepsilon^{2}\left(\partial_{x} h\right)^{2}\right] \partial_{\zeta}^{2} \tilde{r}^{(0)}= & \varepsilon \partial_{x} h\left(\partial_{\zeta} \tilde{A}_{z z}^{(1)}-\partial_{\zeta} \tilde{A}_{x x}^{(1)}\right)+\left[1-\varepsilon^{2}\left(\partial_{x} h\right)^{2}\right] \partial_{\zeta} \tilde{A}_{x z}^{(1)} \\
& +\partial_{x} \tilde{p}^{(0)}-\varepsilon \partial_{x} \tilde{A}_{x x}^{(0)}-\varepsilon^{2} \partial_{x} h \partial_{x} \tilde{A}_{x z}^{(0)}
\end{aligned}
$$

We note that from(A.3d)-(A.3f)

$$
\begin{aligned}
& \varepsilon \partial_{x} h\left(\partial_{\zeta}^{2} \tilde{A}_{z z}^{(1)}-\partial_{\zeta}^{2} \tilde{A}_{x x}^{(1)}\right)+\left(1-\varepsilon^{2}\left(\partial_{x} h\right)^{2}\right) \partial_{\zeta}^{2} \tilde{A}_{x z}^{(1)} \\
& =\frac{\mathrm{De}}{\delta} \frac{1}{1+\varepsilon^{2}\left(\partial_{x} h\right)^{2}}\left[\tilde{A}_{z z}^{(0)}-2 \varepsilon \partial_{x} h \tilde{A}_{x z}^{(0)}+\varepsilon^{2}\left(\partial_{x} h\right)^{2} \tilde{A}_{x x}^{(0)}\right] \partial_{\zeta} \tilde{r}^{(0)}
\end{aligned}
$$

Integration with respect to $\zeta$ and using (A.3j) and (A.4) we obtain the following ordinary differential equation for $\tilde{r}^{0}$ :

$$
\left(1+\varepsilon^{2}\left(\partial_{x} h\right)^{2}\right)\left(2+\varepsilon^{2}\left(\partial_{x} h\right)^{2}\right) \partial_{\zeta}^{2} \tilde{r}^{(0)}=\left(\tilde{A}_{z z}^{(0)}-2 \varepsilon \partial_{x} h \tilde{A}_{x z}^{(0)}+\varepsilon^{2}\left(\partial_{x} h\right)^{2} \tilde{A}_{x x}^{(0)}\right) \tilde{r}^{(0)}+f(x, t),
$$

where

$$
\begin{aligned}
f(x, t)= & \frac{\delta}{\operatorname{De}}\left(1+\varepsilon^{2}\left(\partial_{x} h\right)^{2}\right)\left(\partial_{x} \tilde{p}^{(0)}-\varepsilon \partial_{x} \tilde{A}_{x x}^{(0)}-\varepsilon^{2} \partial_{x} h \partial_{x} \tilde{A}_{x z}^{(0)}\right) \\
& -\left[\tilde{A}_{z z}^{(0)}-2 \varepsilon \partial_{x} h \tilde{A}_{x z}^{(0)}+\varepsilon^{2}\left(\partial_{x} h\right)^{2} \tilde{A}_{x x}^{(0)}\right] \tilde{r}_{\mid \zeta=0}^{(0)} \\
& -\frac{\delta}{\operatorname{De}}\left\{\varepsilon^{4}\left(\partial_{x} h\right)^{2}\left(\partial_{x} \tilde{A}_{z z}^{(0)}-\partial_{x} \tilde{A}_{x x}^{(0)}\right)+\varepsilon^{3} \partial_{x} h\left[1-\varepsilon^{2}\left(\partial_{x} h\right)^{2}\right] \partial_{x} \tilde{A}_{x z}^{(0)}\right\} .
\end{aligned}
$$

The general solution to the second order linear differential equation (A.6) is composed of a particular solution,

$$
\tilde{r}_{p}^{(0)}=-\frac{f(x, t)}{\tilde{A}_{z z}^{(0)}-2 \varepsilon \partial_{x} h \tilde{A}_{x z}^{(0)}+\varepsilon^{2}\left(\partial_{x} h\right)^{2} \tilde{A}_{x x}^{(0)}}
$$


and an exponentially growing and an exponentially decaying complementary solution, provided that

$$
\tilde{A}_{z z}^{(0)}-2 \varepsilon \partial_{x} h \tilde{A}_{x z}^{(0)}+\varepsilon^{2}\left(\partial_{x} h\right)^{2} \tilde{A}_{x x}^{(0)}>0 .
$$

(This is satisfied if we restrict our attention to flows that do not deviate much from channel flow, so that $\tilde{A}_{z z}^{(0)} \sim 1$ and $\varepsilon$ is small.) The exponentially growing solution is not matchable, and hence that contribution has been eliminated. Then, for $\zeta \rightarrow \infty, \tilde{r}^{0}$ tends to $r_{p}^{(0)}$, so we match this to the combination $u^{(0)}+\varepsilon^{2} \partial_{x} h w^{(0)}$ of the leading order outer solutions. The matching condition can then be solved for $f(x, t)$ and from this and (A.7) we obtain $\left.\tilde{r}^{(0)}\right|_{\zeta=0}$ in terms of the outer solutions. Now we can integrate (A.1f) once with respect to $\zeta$ and then use (A.1j) as well as the information about $\left.\tilde{r}^{(0)}\right|_{\zeta=0}$ we just obtained to fix the integration constants. The resulting expression

$$
\partial_{\zeta} \tilde{A}_{z z}^{(1)}=\varepsilon^{2} \times O(1) \text { terms }
$$

can be matched to the outer solution, which gives a Neumann condition for the outer $A_{z z}$,

$$
\left.\partial_{z} A_{z z}^{(0)}\right|_{z=h}=\varepsilon^{2} \times O(1) \text { terms }
$$

with a right hand side that (after matching) only depends on leading order variables of the outer solution. Here, we do not need the precise form of the right hand side as our goal is to justify (5.11). Indeed, (A.9) reduces to (5.11) in the limit $\varepsilon \rightarrow 0$.

\section{References.}

[1] N. P. Adhikari and J. L. Goveas, Effects of slip on the viscosity of polymer melts, J. Polymer Sci.: Part B: Polymer Physics, 42 (2004), pp. 1888-1904.

[2] A. AJDARI, Slippage at a polymer/polymer interface: Entanglements and associated friction, C.R. Acad. Sci., Ser. II, 317 (1993), pp. 1159-1163.

[3] A. Ajdari, F. B. Wyart, P. G. De Gennes, L. Leibler, J. Viovy, and M. Rubinstein, Slippage of an entangled polymer melt on a grafted surface, Physica A, 204 (1994), pp. 17-39.

[4] P. Ballesta, G. Petekidis, L. Isa, W. C. K. Poon, and R. Besseling, Wall slip and flow of concentrated hard-sphere colloidal suspensions, J. Rheol., 56 (2012), p. 1005.

[5] A. Bhardwaj, E. Miller, and J. Rothstein, Filament stretching and capillary breakup extensional rheometry measurements of viscoelastic wormlike micelle solutions, J. Rheol., 51 (2007), pp. 693-719.

[6] F. Brochard-Wyart and P. De Gennes, Shear-dependent slippage at a polymer/solid interface, Langmuir, 8 (1992), pp. 3033-3037.

[7] F. Brochard-Wyart and P. De Gennes, Sliding molecules at a polymer/polymer interface, C. R. Acad. Sci. Ser. II, 327 (1993), pp. 13-17.

[8] M. Cromer, L. P. Cook, And F. MCKinley, Pressure-driven flow of wormlike micellar solutions in rectilinear microchannels, J. of Non-Newtonian Fluid Mech., 166 (2011), pp. 180-193.

[9] A. El-Kareh and G. Leal, The existence of solutions for all Deborah numbers for non-Newtonian fluids, J. Non-Newtonian Fluid Mech., 33 (1989), pp. 257-287.

[10] R. Fetzer, K. Jacobs, A. Münch, B. Wagner, and T. P. Witelski., New slip regimes and the shape of dewetting thin liquid films., Phys. Rev. Lett., 95 (2005), p. 127801.

[11] S. Fielding, Complex dynamics of shear banded flows, Soft Matter, 2 (2007), pp. 1262-1279.

[12] H. P. Greenspan, On the motion of a small viscous droplet that wets a surface, Journal of Fluid Mechanics, 84 (1978), pp. 125-143.

[13] C. Huh And L. Scriven, Hydrodynamic model of steady movement of a solid/liquid/fluid contact line, Journal of Colloid and Interface Science, 35 (1971), pp. 85-101.

[14] S. Jachalski, A. MÜNCh, AND B. WaGneR, Thin-film models for viscoelastic liquid bi-layers. WIAS Preprint 2187, 2015.

[15] S. M. L. BÉCu And A. Colin, Spatiotemporal dynamics of wormlike micelles under shear, Phys. Rev. Lett., 93 (2004), p. 018301.

[16] E. Lauga, M.P.Brenner, and H. Stone, Microfluidics: The no-slip boundary condition, in Handbook of Experimental Fluid Dynamics, Springer, New York, 2007, pp. 1219-1240.

[17] L. LÉGER, Friction mechanisms and interfacial slip at fluid-solid interfaces, J. Phys.: Condensed Matter, 15 (2003), pp. S19-S29. 
[18] M. P. Lettinga And S. Manneville, Competition between shear banding and wall slip in wormlike micelles, Phys. Rev. Lett., 103 (2009), p. 248302.

[19] C. Masselon, A. Colin, And P. D. Olmsted, Influence of boundary conditions and confinement on nonlocal effects in flows of wormlike micellar systems, Physical Review E, 81 (2010), p. 021502.

[20] A. MÜnch And B. Wagner, Contact-line instability of dewetting thin films, Physica D, 209 (2005), pp. $178-190$.

[21] A. Münch, B. Wagner, M. Rauscher, and R. Blossey, A thin-film model for corotational Jeffreys fluids under strong slip, The European Physical Journal E, 20 (2006), pp. 365-368.

[22] A. Münch, B. Wagner, And T. P. Witelski, Lubrication models with small to large slip lengths, J. Engr. Math., 53 (2006), pp. 359-383.

[23] P. Neogi And C. A. Miller, Spreading kinetics of a drop on a rough solid surface, Journal of Colloid and Interface Science, 92 (1983), pp. 338-349.

[24] P. Olmsted, Perspectives on shear banding in complex fluids, Rheol. Acta, 47 (2008), pp. 283-300.

[25] S. E. Orchard, On surface leveling in viscous liquids and gels, Appl. Sci. Res. A, 11 (1962), pp. 451-464.

[26] C. L. P. Olmsted, O. RAdulescu, Johnson-segalman model with a diffusion term in cylindrical couette flow, J. Rheol., 44 (2000), pp. 257-275.

[27] P. A. Vasquez, G. H. McKinley, and P. L. Cook, A network scission model for wormlike micellar solutions, Journal of Non-Newtonian Fluid Mechanics, 144 (2007), pp. 122-139.

[28] L. Zhou, P. Vasquez, L. Cook, And G. H. McKinley, Modeling the inhomogeneous response and formation of shear bands in steady and transient flows of entangled fluids, J. Rheol., 32 (2008), pp. 591623. 